\title{
SMALL DEGREE REPRESENTATIONS OF FINITE CHEVALLEY GROUPS IN DEFINING CHARACTERISTIC
}

\author{
FRANK LÜBECK
}

\begin{abstract}
The author has determined, for all simple simply connected reductive linear algebraic groups defined over a finite field, all the irreducible representations in their defining characteristic of degree below some bound. These also give the small degree projective representations in defining characteristic for the corresponding finite simple groups. For large rank $l$, this bound is proportional to $l^{3}$, and for rank less than or equal to 11 much higher. The small rank cases are based on extensive computer calculations.
\end{abstract}

\section{Introduction}

In this paper we give lists of projective representations of simple Chevalley groups in their defining characteristic. There are two types of results.

First, we determine for groups of rank less than or equal to 11, all such representations of degree less than or equal to some bound depending on the type (for example, 100000 for type $E_{8}$ ); see Theorem 4.4 for details. These data were produced using a collection of computer programs developed by the author. Of course, some of the degrees given in our tables had been known previously. To give two examples: Gilkey and Seitz handle some cases for exceptional types with computational methods in [6]. Some small rank cases, such as types $B_{2}$ and $G_{2}$, are handled more theoretically by Jantzen in [11] (although the explicit degrees are still not particularly easy to obtain from that result).

Secondly, we determine for groups of classical type with rank $l$, all representations of degree at most $l^{3} / 8$ for type $A_{l}$, and of degree at most $l^{3}$ for the other types. For large $l$ this is a small list, given in Theorem 5.1, and for small $l$ this range is easily covered by the tables mentioned above. This extends similar results by Liebeck with bounds proportional to $l^{2}$; see $[13,5.4 .11]$ and the references given there.

Our results contain in particular a complement to the tables of representations in nondefining characteristic up to degree 250, worked out by Hiß and Malle, and given in [7].

We fix some notation for the whole paper. Let $\tilde{G}$ be a finite twisted or non-twisted simple Chevalley group in characteristic $p$ (we consider ${ }^{2} F(2)^{\prime}$ as sporadic and exclude it here, but see the corresponding remark after Theorem 4.4).

There is an associated connected reductive simple algebraic group $G$ over $\overline{\mathbb{F}}_{p}$ of simply connected type, a Frobenius endomorphism $F$ of $G$, a number $q=p^{f}$ with $2 f \in \mathbb{Z}$, and an $m \in \mathbb{N}$ such that:

- $G$ is defined over $\mathbb{F}_{q^{m}}$ via $F^{m}$;

- for the group of $F$-fixed points $G(q)$ with center $Z$ we have $\tilde{G} \cong G(q) / Z$;

- $G(q)$ is the quotient of the universal covering group of $\tilde{G}$ by the $p$-part of its center. 
(Note that this includes the Suzuki and Ree groups.)

So, asking for the projective representations of $\tilde{G}$ in characteristic $p$ is the same as asking for the representations of $G(q)$ in characteristic $p$. These can be constructed by restricting certain representations, called the highest-weight representations, of the algebraic group $G$ to $G(q)$. This is explained in Section 2. In Section 3 we briefly describe how our computer programs for computing weight multiplicities work. In Section 4 we describe our main result, consisting of lists of small degree representations for groups of rank at most 11 (the lists themselves are printed in Appendix A). Finally, in Section 5 we consider groups of larger rank.

\section{Representations in defining characteristic}

There are several highly readable introductions to this topic, for example Humphreys' survey [9]. A detailed reference is Jantzen's book [12]. We recall some of the basic facts.

For a group $G$ of simply connected type and of $\operatorname{rank} l$, as in the introduction, let $T$ be a maximal torus of $G$, let $X \cong \mathbb{Z}^{l}$ be its character group, and let $Y \cong \mathbb{Z}^{l}$ be its co-character group.

Let $\left\{\alpha_{1}, \ldots, \alpha_{l}\right\} \subset X$ be a set of simple roots for $G$ with respect to $T$, and let $\alpha_{i}^{\vee} \in Y$ be the coroot corresponding to $\alpha_{i}$, for $i=1, \ldots, l$. Viewing $X \otimes \mathbb{R}$ as Euclidean space, we define the fundamental weights $\omega_{1}, \ldots, \omega_{l} \in X \otimes \mathbb{R}$ as the dual basis of $\alpha_{1}^{\vee}, \ldots, \alpha_{l}^{\vee}$. This is a $\mathbb{Z}$-basis of $X$ (because $G$ is simply connected).

There is a partial ordering $\leqslant$ on $X$, defined by $\omega \leqslant \omega^{\prime}$ if and only if $\omega^{\prime}-\omega$ is a nonnegative linear combination of simple roots. A weight $\omega \in X$ is called dominant if it is a non-negative linear combination of the fundamental weights. The Weyl group $W$ of $G$, generated by the reflections along the $\alpha_{i}$, acts on $X$. Under this action, each $W$-orbit on $X$ contains a unique dominant weight.

From now on, let $L$ be a finite-dimensional $G$-module over $\overline{\mathbb{F}}_{p}$. If this is considered as a $T$-module, there is a direct sum decomposition $L=\bigoplus_{\omega \in X} L_{\omega}$ into weight spaces $L_{\omega}$ such that $t \in T$ acts by multiplication with $\omega(t)$ on $L_{\omega}$. The set of $\omega \in X$ with $L_{\omega} \neq\{0\}$ is called the set of weights of $L$. The set of weights of $L$ is a union of $W$-orbits, and for $\omega \in X, x \in W$ we have $\operatorname{dim}\left(L_{\omega}\right)=\operatorname{dim}\left(L_{\omega x}\right)$.

The following basic results characterize the irreducible representations $L$ via their set of weights.

Theorem 2.1 (Chevalley). Let $G$ and $L$ be as above.

(a) If $L$ is irreducible, then the set of weights of $L$ contains a (unique) element $\lambda$ such that for all weights $\omega$ of $L$, we have $\omega \leqslant \lambda$. This $\lambda$ is called the highest weight of $L$, it is dominant, and we have $\operatorname{dim}\left(L_{\lambda}\right)=1$.

(b) An irreducible $G$-module L is determined up to isomorphism by its highest weight.

(c) For each dominant weight $\lambda \in X$, there is an irreducible $G$-module $L(\lambda)$ with highest weight $\lambda$.

A dominant weight $\lambda=a_{1} \omega_{1}+\cdots+a_{l} \omega_{l} \in X$ is called $p$-restricted if $0 \leqslant a_{i} \leqslant p-1$ for $1 \leqslant i \leqslant l$.

The following result of Steinberg shows how all highest-weight modules $L(\lambda)$ of $G$ can be constructed out of those with $p$-restricted highest weights. 
Theorem 2.2 (Steinberg's tensor product theorem). Let $F_{0}$ be the Frobenius automorphism of $\overline{\mathbb{F}}_{p}$, raising elements to their pth power. Twisting the $G$-action on a $G$-module $L$ with $F_{0}^{i}, i \in \mathbb{Z}_{\geqslant 0}$, we get another $G$-module, which we denote by $L^{(i)}$. If $\lambda_{0}, \ldots, \lambda_{n}$ are p-restricted weights, then

$$
L\left(\lambda_{0}+p \lambda_{1}+\cdots+p^{n} \lambda_{n}\right) \cong L\left(\lambda_{0}\right) \otimes L\left(\lambda_{1}\right)^{(1)} \otimes \cdots \otimes L\left(\lambda_{n}\right)^{(n)} .
$$

Finally, we need to recall the relation between the irreducible modules of the algebraic group $G$ and those of the finite group $G(q)$. This is nicely described by Steinberg in $[19,13.3,11.6]$.

Theorem 2.3 (Steinberg). Let $G$ and $G(q)$ be as in the introduction. We define a subset $\Lambda$ of dominant weights. If $G(q)$ is not a Suzuki or Ree group (that is, not of type ${ }^{2} B_{2},{ }^{2} G_{2}$ or ${ }^{2} F_{4}$ ), then $\Lambda=\left\{a_{1} \omega_{1}+\cdots+a_{l} \omega_{l} \mid 0 \leqslant a_{i} \leqslant q-1\right.$ for $\left.1 \leqslant i \leqslant l\right\}$. In the case of Suzuki and Ree groups, we define

$$
\begin{aligned}
\Lambda= & \left\{a_{1} \omega_{1}+\cdots+a_{l} \omega_{l} \mid\right. \\
& \left.0 \leqslant a_{i} \leqslant q / \sqrt{p}-1 \text { if } \alpha_{i} \text { is a long root, } 0 \leqslant a_{i} \leqslant q \sqrt{p}-1 \text { if } \alpha_{i} \text { is a short root }\right\} .
\end{aligned}
$$

(Note that $q$ is the square root of an odd power of $p=2,3$ or $p=2$, respectively, in these cases.)

Then the restrictions of the $G$-modules $L(\lambda)$ with $\lambda \in \Lambda$ to $G(q)$ form a set of pairwise inequivalent representatives of all equivalence classes of irreducible $\overline{\mathbb{F}}_{p} G(q)$-modules.

These results show that the dimensions of the irreducible representations of the groups $G(q)$ over $\overline{\mathbb{F}}_{p}$ are easy to obtain if we know the dimensions of the representations $L(\lambda)$ of the algebraic groups $G$ for $p$-restricted weights $\lambda$.

\section{Computation of weight multiplicities}

In this section we sketch how we compute the degree of the representation $L(\lambda)$ for a given root datum of $G$, highest weight $\lambda$ and prime $p$. For fixed $G$ and $\lambda$, the degree of $L(\lambda)$ is the same for almost all $p$; namely, it is the degree of $L(\lambda)$ for the algebraic group over the complex numbers with the same root datum, or for its Lie algebra. In these cases the degree can be computed by a formula of Weyl; see [8, 24.3].

In the other cases, no formula is known. There is, however, in principle an algorithm to compute the degree. This is described in [8, Exercise 2 of 26.4], and goes back to work by Burgoyne [2]. This was also used in [6] to handle some cases in exceptional groups. The idea is to construct a so-called 'Weyl module' $V(\lambda)_{\mathbb{Z}}$ generically over the integers. By a base change, this leads to a module $V(\lambda)$ for any $G$ with the given root datum over any ring, which has $\lambda$ as a highest weight. Over $\mathbb{C}$ or over $\overline{\mathbb{F}}_{p}$ for almost all $p$, this is irreducible and thus isomorphic to $L(\lambda)$. In general, $L(\lambda)$ is a quotient of $V(\lambda)$.

To construct $V(\lambda)_{\mathbb{Z}}$, one considers the universal enveloping algebra $U$ of the complex Lie algebra corresponding to the given root datum. It contains a $\mathbb{Z}$-lattice $\mathcal{U}_{\mathbb{Z}}$, the Kostant $\mathbb{Z}$-form of $\mathcal{U}$, which is defined via a Chevalley basis of the Lie algebra. Up to equivalence, there is a unique irreducible highest-weight representation $V(\lambda)$ for $\mathcal{U}$ with highest weight $\lambda$. Let $0 \neq v \in V(\lambda)$ be a vector of weight $\lambda$ (this is unique up to scalar). Then we set $V(\lambda)_{\mathbb{Z}}:=u_{\mathbb{Z}} v=U_{\mathbb{Z}}^{-} v$.

We fix an ordering $\beta_{1}, \ldots, \beta_{N}$ of the set of positive roots. Then $\mathcal{U}_{\mathbb{Z}}^{-}$and $\boldsymbol{U}_{\mathbb{Z}}^{+}$have $\mathbb{Z}$-bases labeled by sequences $\mathbf{a}=\left(a_{1}, \ldots, a_{N}\right)$ of non-negative integers. 
Applying such a basis element $f_{\mathbf{a}}$ of $\mathcal{U}_{\mathbb{Z}}^{-}$to a vector of weight $\omega$, we get a vector of weight $\omega-a_{1} \beta_{1}-\cdots-a_{N} \beta_{N}$ (and similarly for basis vectors $e_{\mathbf{a}}$ of $\mathcal{U}_{\mathbb{Z}}^{+}$). So, decomposing $V(\lambda)_{\mathbb{Z}}=\bigoplus_{\omega \in X}\left(V_{\omega}\right)_{\mathbb{Z}}$ according to the weight spaces, we can describe the generating sets of $\left(V_{\omega}\right)_{\mathbb{Z}}$ by all the non-negative linear combinations of positive roots which are equal to $\lambda-\omega$.

There is a non-degenerate bilinear form $(\cdot, \cdot)$ on $V(\lambda)_{\mathbb{Z}}$ which can be described via this generating system. Different weight spaces are orthogonal with respect to this form. Let a and $\mathbf{b}$ be two coefficient vectors as above, such that the corresponding linear combination of the positive roots is the same. Then $e_{\mathbf{b}} f_{\mathbf{a}} v=n_{\mathbf{a}, \mathbf{b}} v$ is again of weight $\lambda$, and one defines $\left(f_{\mathbf{a}} v, f_{\mathbf{b}} v\right):=n_{\mathbf{a}, \mathbf{b}} \in \mathbb{Z}$.

Since the form is nondegenerate, we can compute the rank of a weight lattice $\left(V_{\omega}\right)_{\mathbb{Z}}$ by computing the rank of the matrix $\left(n_{\mathbf{a}, \mathbf{b}}\right)$, where $\mathbf{a}$ and $\mathbf{b}$ are running through all the non-negative linear combinations of positive roots for $\lambda-\omega$. The dimension of the weight space $L_{\omega}$ of $L(\lambda)$ is the rank of $\left(n_{\mathbf{a}, \mathbf{b}}\right)$ modulo $p$.

The coefficients $n_{\mathbf{a}, \mathbf{b}}$ can be computed by simplifying the element $e_{\mathbf{b}} f_{\mathbf{a}}$ with the help of the commutator relations in $\mathcal{U}$; see $[8,25]$. These involve the structure constants for a Chevalley basis of the corresponding Lie algebra, which can be computed as described in $[3,4.2]$.

In principle, this allows the determination of $\operatorname{dim}(L(\lambda))$ for all $G, p$ and $\lambda$, but in practice these computations can become very long, even in small examples. There are technical problems such as the question of how to apply commutator relations most efficiently in order to compute the integers $n_{\mathbf{a}, \mathbf{b}}$. The use of different strategies may considerably alter the number of steps in the calculation. However, the main problem is that the generating sets for the weight spaces as described above are highly redundant. Usually, the number of non-negative linear combinations of positive roots which yield $\lambda-\omega$ is much larger than the dimension of $V_{\omega}$. By a careful choice of the ordering of the positive roots, one can reduce the linear combinations to be considered, because for many $\mathbf{a}=\left(a_{1}, \ldots, a_{N}\right)$ with $\omega=\lambda-a_{1} \beta_{1}-\cdots-a_{N} \beta_{N}$ there is a $1 \leqslant k \leqslant N$ such that $\lambda-a_{1} \beta_{1}-\cdots-a_{k} \beta_{k}$ is not a weight of $V(\lambda)$, and hence $f_{\mathbf{a}} v=0$.

However, the main improvement that we get is achieved by using results of Jantzen and Andersen; see [12, II.8.19]. The so-called 'Jantzen sum formula' expresses the determinant of the Gram matrix of the bilinear form $(\cdot, \cdot)$ on the lattice $\left(V_{\omega}\right)_{\mathbb{Z}}$ in terms of weight multiplicities of various $V(\mu)$ with $\mu \leqslant \lambda$. The weight multiplicities of these $V(\mu)$ can be efficiently computed by Freudenthal's formula; see [8, 22.3]. In particular, the formula gives exactly the set of primes $p$ for which the Weyl module $V(\lambda)$ is not isomorphic to $L(\lambda)$. In rare cases, it happens that a prime $p$ divides such a determinant exactly once-and then we know without further calculations that $\operatorname{dim}\left(L_{\omega}\right)=\operatorname{dim}\left(V_{\omega}\right)-1$.

Using these determinants we compute only parts of the matrices $\left(n_{\mathbf{a}, \mathbf{b}}\right)$ corresponding to a subset of its rows and columns until the submatrix has the full rank $\operatorname{dim}\left(V_{\omega}\right)$ and the product of its elementary divisors is equal to the known determinant. Then the rank of $\left(n_{\mathbf{a}, \mathbf{b}}\right)$ modulo $p$ is the same as the rank of this submatrix modulo $p$.

With this approach, we never need submatrices of $\left(n_{\mathbf{a}, \mathbf{b}}\right)$ of much larger dimension than $\operatorname{dim}\left(V_{\omega}\right)$. (In [6], consideration of the matrices $\left(n_{\mathbf{a}, \mathbf{b}}\right)$ was replaced by computing somewhat smaller matrices using the action of parabolic subalgebras in cases where $\lambda$ has a non-trivial stabilizer in $W$, but these matrices were still big compared to the dimension of the weight spaces.) Of course, our method is limited to representations where no single weight space has a dimension of more than a few thousand.

Actually, by using our approach we can also compute the Jantzen filtrations of the Weyl 
modules (see [12, II.8]), by computing the elementary divisors of the matrices $\left(n_{\mathbf{a}, \mathbf{b}}\right)$ and not just their rank. One needs a sophisticated algorithm to compute the exact elementary divisors of such matrices, of dimension bigger than 200, say. We developed the algorithm described in [14] for this purpose.

We have a collection of computer programs for doing the calculations described above; they are based on the computer algebra system GAP [17] and the package CHEVIE [5].

It is planned to make them available to other users in the form of another package. This will be built on a new version of CHEVIE for GAP - Version 4, which is currently being developed. While rewriting the current programs, we hope to improve their efficiency, and to extend their functionality. We postpone the publication of a much more detailed version of this very sketchy section until this package is ready.

\section{Representations of small degree for groups of small rank}

From Theorem 2.2, we see how to construct any highest-weight representation $L(\lambda)$ of $G$ from those with $p$-restricted weights by twisting with field automorphisms and tensoring.

Assume now that we are given the type of an irreducible root system, and a number $M \in \mathbb{N}$. We consider the groups $G$ over $\overline{\mathbb{F}}_{p}$ with this root system, for all $p$ at once. We want to find, for all primes $p$, all the $p$-restricted dominant weights $\lambda$ such that the highest-weight representation $L(\lambda)$ of the group $G$ over $\overline{\mathbb{F}}_{p}$ has degree smaller than or equal to $M$.

Our main tool in restricting this question to the consideration of a finite number of $\lambda$ is the following result by Premet; see [16]. Recall from Section 2 that the set of weights of $L(\lambda)$ is a union of $W$-orbits; and that each $W$-orbit contains a unique representative which is dominant.

Theorem 4.1 (Premet). If the root system of $G$ has different root lengths, we assume that $p \neq 2$, and if $G$ is of type $G_{2}$, we also assume that $p \neq 3$. Let $\lambda$ be a $p$-restricted dominant weight. Then the set of weights of $L(\lambda)$ is the union of the $W$-orbits of dominant weights $\omega$ with $\omega \leqslant \lambda$.

We note that in the exceptional cases with different root lengths and either $p=2$ or $p=3$, the statement of the theorem does not actually hold.

The following remark shows how to compute explicitly the number of weights of $L(\lambda)$ as given by Premet's theorem.

Remark 4.2. (a) Let $\omega=a_{1} \omega_{1}+\cdots+a_{l} \omega_{l}$ be a dominant weight. Then the stabilizer of $\omega$ in the Weyl group $W$ is the parabolic subgroup generated by the reflections along the simple roots $\alpha_{i}$ for which $a_{i}=0$.

(b) For given dominant weight $\lambda$, one finds the set $\Omega=\{\omega \mid \omega$ dominant and $\omega \leqslant \lambda\}$ efficiently as follows. Initialize $\Omega \leftarrow\{\lambda\}$. For each $\omega \in \Omega$ and for each positive root $\alpha$, compute $\omega-\alpha$. If this is also dominant, add it to the set $\Omega$.

Proof. For part (a) see [8, 10.3B], and for part (b) see [15, Proposition 1].

Here is an algorithm for finding a set of candidate highest weights for representations of $G$ of rank at most a given bound. It shows in particular that, for any given bound, there is only a finite number of highest weights that must be considered. 
Algorithm 4.3. Input: $A$ root system and an $M \in \mathbb{N}$.

Output: A set A of dominant weights which contains all $\lambda$ such that there is a prime $p$ for which $\lambda$ is p-restricted, and a group $G$ over $\overline{\mathbb{F}}_{p}$ corresponding to the given root system with highest-weight representation $L(\lambda)$ of degree at most $M$.

(1) For a dominant weight $\lambda$, we define $m(\lambda)$ as the number of weights in all the $W$-orbits of dominant weights $\omega$ with $\omega \leqslant \lambda$. The numbers $m(\lambda)$ can be computed using Remark 4.2.

(2) We initialize the set $A$ with all 2-restricted weights $\lambda$ with $m(\lambda) \leqslant M$.

(3) We choose a linear function $\gamma: X \rightarrow \mathbb{Z}$ which takes positive values on the simple roots $\alpha_{i}$, where $1 \leqslant i \leqslant l$ (and hence on the fundamental weights).

Then we start to enumerate recursively, for $n=0,1, \ldots$, all the dominant weights $\lambda$ with $\gamma(\lambda)=n$. (Clearly, for fixed $n$ there is a finite number of such weights.) For each of these $\lambda$ we compute $m(\lambda)$, and if it is less than or equal to $M$, we put $\lambda$ into $A$.

We proceed until we find an interval $I=\left[a, a+\max \left(\gamma\left(\alpha_{i}\right) \mid 1 \leqslant i \leqslant l\right)\right]$ such that $a>\gamma(\lambda)$ for all $\lambda \in A$, and such that for all dominant $\lambda$ with $\gamma(\lambda) \in I$ we have $m(\lambda)>M$.

(4) If the given root system has roots of different length, we add all the 2-restricted weights to A. If there is a component of type $G_{2}$, we also add all the 3-restricted weights.

(5) We return the set $A$.

Proof. We have to show that we will eventually find an interval $I$, as described in step (3), and that all the dominant weights that have not been considered during the algorithm lead to modules of dimension greater than $M$.

In the cases of root systems with different root lengths and $p=2$ or $p=3$, for which Premet's theorem 4.1 does not give a statement, we have put all the 2-and 3-restricted weights, respectively, into $A$ in step (4). So, from now on we consider only the weights and primes for which Premet's theorem is applicable. This theorem says that $m(\lambda)$, defined in (1), is a lower bound for $\operatorname{dim}(L(\lambda))$ for all primes $p$ such that $\lambda$ is $p$-restricted.

Now we show that in steps (2) and (3) we construct the set $A$ as the set of dominant weights $\lambda$ with $m(\lambda) \leqslant M$.

First note that for $\omega \leqslant \lambda$ dominant, we have $m(\omega) \leqslant m(\lambda)$.

Furthermore, let $\lambda=a_{1} \omega_{1}+\cdots+a_{l} \omega_{l}$ be a dominant weight which is not 2-restricted; that is, one where $a_{i} \geqslant 2$. Then $\lambda-\alpha_{i}$ is also a dominant weight. This follows from the fact that the coefficients of the simple root $\alpha_{i}$, expressed as a linear combination of the fundamental weights, is given by the $i$ th column of the Cartan matrix (which has entries of 2 on the diagonal, and non-positive entries elsewhere).

So, for a weight $\lambda$ with one of the coefficients $a_{i} \geqslant 2 k$, where $k \in \mathbb{N}$, there are at least $k$ smaller dominant weights, and so $m(\lambda)>k$. This shows that we are constructing a finite set, and that we will find the interval $I$ in step (3).

Now we show by induction on the value $n$ of $\gamma$, starting from the lower bound $n=a$ of the interval $I$, that for a dominant weight $\lambda$ with $\gamma(\lambda)=n$, we have $m(\lambda)>M$.

This is clear for $n \in I$, by the construction of the interval $I$ in step (3).

So, assume that $\gamma(\lambda)>a+\max \left(\gamma\left(\alpha_{i}\right) \mid 1 \leqslant i \leqslant l\right)$. If $\lambda$ is 2-restricted, we have found in step (2) that $m(\lambda)>M$. (It is not in $A$, by the construction of $I$.) Otherwise, one coefficient of $\lambda$, say $a_{i}$, is greater than or equal to 2 . Then $\lambda-\alpha_{i}$ is dominant, and we have $a \leqslant \gamma\left(\lambda-\alpha_{i}\right)<\gamma(\lambda)$. By the induction hypothesis, we get $M<m\left(\lambda-\alpha_{i}\right)<m(\lambda)$.

In Table 1 we fix some bound $M$ for each irreducible root system of rank at most 11 , or rank 17 in the case $A_{l}$. 
Small degree representations in defining characteristic

Table 1: Chosen degree bounds for groups of small rank

\begin{tabular}{|c|c|c|c|c|c|c|c|c|c|c|}
\hline Type & $A_{2}$ & $A_{3}$ & $A_{4}$ & $A_{5}$ & $A_{6}$ & $A_{7}$ & $A_{8}$ & $A_{9}$ & $A_{10}$ & $A_{11}$ \\
\hline $\mathrm{M}$ & 400 & 500 & 1000 & 2500 & 2800 & 3000 & 4000 & 6000 & 10000 & 12000 \\
\hline Type & $A_{12}$ & $A_{13}$ & $A_{14}$ & $A_{15}$ & $A_{16}$ & $A_{17}$ & & & & \\
\hline M & 3000 & 3000 & 3000 & 3000 & 3000 & 3000 & & & & \\
\hline Type & $B_{2}$ & $B_{3}$ & $B_{4}$ & $B_{5}$ & $B_{6}$ & $B_{7}$ & $B_{8}$ & $B_{9}$ & $B_{10}$ & $B_{11}$ \\
\hline M & 300 & 700 & 1000 & 2000 & 4000 & 5000 & 7000 & 8000 & 10000 & 12000 \\
\hline Type & & $C_{3}$ & $C_{4}$ & $C_{5}$ & $C_{6}$ & $C_{7}$ & $C_{8}$ & $C_{9}$ & $C_{10}$ & $C_{11}$ \\
\hline M & & 1000 & 2000 & 2500 & 4000 & 6000 & 10000 & 10000 & 10000 & 12000 \\
\hline Type & & & $D_{4}$ & $D_{5}$ & $D_{6}$ & $D_{7}$ & $D_{8}$ & $D_{9}$ & $D_{10}$ & $D_{11}$ \\
\hline M & & & 2000 & 3000 & 4000 & 5000 & 10000 & 15000 & 18000 & 20000 \\
\hline Type & $G_{2}$ & & $F_{4}$ & & $E_{6}$ & $E_{7}$ & $E_{8}$ & & & \\
\hline M & 500 & & 12000 & & 50000 & 100000 & 100000 & & & \\
\hline
\end{tabular}

For each irreducible root system listed in Table 1, we used the given $M$ as input for Algorithm 4.3. For each weight $\lambda$ in the output set $A$, and for all primes $p$ for which $\lambda$ is $p$-restricted, we computed the exact weight multiplicities of $L(\lambda)$, using the techniques described in Section 3.

We remark that the sets $A$ obtained from Algorithm 4.3 are much larger than the list of weights that actually lead to representations of dimension less than or equal to $M$, since we used a very rough lower bound for these dimensions. For weights of representations of much larger degree, however, we usually find, quite quickly, some weight multiplicities greater than 1, which gives better lower bounds for the dimension. We stop the computation of weight multiplicities when this better estimate becomes larger than $M$. In this way, almost all of the computation time required to obtain our result was actually spent on those weights that appear in our result tables.

The bounds $M$ were chosen such that the results presented below could be computed interactively, using our programs, within about two days, employing ten computers in parallel. The types $A_{l}$, for $12 \leqslant l \leqslant 17$, were added at the request of Gunter Malle, who asked us to cover all representations of degree less than or equal to $2 \operatorname{dim}(G)$ in this note, for a specific application.

Here is our main result.

Theorem 4.4. For any type of root system and number $M$ as given in Table 1, and all primes $p$, the tables in Appendix A.6 to Appendix A.53 list the p-restricted weights $\lambda$ such that the representation $L(\lambda)$ of the algebraic group $G$ over $\overline{\mathbb{F}}_{p}$ has degree at most $M$. The exact degree of $L(\lambda)$ is also given. Furthermore, we describe the centers of the groups $G$ and the action of the fundamental weights on the center in Appendix A.2. This allows us to determine the kernels of the representations $L(\lambda)$. The Frobenius-Schur indicators in the case where $p \neq 2$ are given by Appendix A.3.

As mentioned above, we have actually computed the exact weight multiplicities for the representations appearing in the tables of Appendix A. It would take too much space to print these in detail, but the results are available upon request from the author.

Our table for type $F_{4}$ includes a description of the representations of the Ree group ${ }^{2} F_{4}(2)$. The related finite simple group is the commutator subgroup which is of index 2. 
The restrictions of the irreducible representations of ${ }^{2} F_{4}(2)$ in characteristic 2 remain irreducible, except for that with highest weight $\lambda=(1100)$, which splits into two representations of degree 2048; see the Modular Atlas [10].

The types considered above do not include $A_{1}$; that is, $G=\mathrm{SL}_{2}\left(\overline{\mathbb{F}}_{p}\right)$. The reason is that this case is easy to describe systematically. This seems to be well known, but also follows immediately from Theorem 4.1, since all the weight multiplicities are at most 1 in this case; see $[8,7.2]$.

Remark 4.5. If $G$ is of type $A_{1}$, then the representation $L\left(k \omega_{1}\right)$ with $0 \leqslant k \leqslant p-1$ has degree $k+1$. For odd $p$, the center of $G$ is non-trivial and of order 2 . It is contained in the kernel of $L\left(k \omega_{1}\right)$ if and only if $k$ is even.

\section{Representations of small degree for groups of large rank}

In this section we consider classical groups of large rank. We prove the following result.

Theorem 5.1. Let $G$ be of classical type, and let $l$ be the rank of $G$. Set $M=l^{3} / 8$ if $G$ is of type $A_{l}$, and $M=l^{3}$ otherwise. If $l>11$, then all $p$-restricted weights $\lambda$ such that the highest-weight representation $L(\lambda)$ of $G$ has dimension at most $M$ are given in Table 2 . The table also includes the dimensions of these modules. (Note that the case of type $B_{l}$ and $p=2$ is included in the case $C_{l}$ and $p=2$.)

The fundamental weights are labeled as explained in Appendix A.1.

Note that the corresponding result for $G$ of $\operatorname{rank} l \leqslant 11$ is included in Theorem 4.4.

Proof. We first prove that the weights which do not appear in our table correspond to representations of degree larger than $M$.

(Case $\left.B_{l}, C_{l}\right) \quad$ Let $\lambda=a_{1} \omega_{1}+\cdots+a_{l} \omega_{l}$ be a dominant weight. If some $a_{i} \neq 0$, then the $W$-stabilizer of $\lambda$ is contained in a reflection subgroup of type $B_{i-1} \times A_{l-i}$; see Remark 4.2. Hence the $W$-orbit of $\lambda$, and so $\operatorname{dim}(L(\lambda))$, is at least

$$
b_{i}:=\left(2^{l} l !\right) /\left(2^{i-1}(i-1) ! \cdot(l-i+1) !\right)=2^{l-i+1}\left(\begin{array}{c}
l \\
i-1
\end{array}\right) .
$$

For $l \geqslant 12$ and $1 \leqslant i \leqslant l-2$, we have $b_{i}>l^{3}$. If $a_{l-1} \neq 0$ and $a_{l} \neq 0$, then the stabilizer of $\lambda$ is contained in a reflection group of type $B_{l-2}$, whose index is greater than $l^{3}$ for all $l \geqslant 6$.

This shows that only weights with at most one non-zero coefficient, either $a_{l-1}$ or $a_{l}$, can appear in our list. If $a_{l-1} \geqslant 2$, then, as explained in the proof of Algorithm $4.3, \lambda-\alpha_{l-1} \leqslant \lambda$ is also a dominant weight. But this has coefficient 1 at $\omega_{l-2}$. Using the estimate as above for this smaller weight, and Premet's theorem 4.1 (note that the $\lambda$ considered now is not 2 -restricted), we see again that $\operatorname{dim}(L(\lambda))>l^{3}$. A similar argument shows that $a_{l}<3$ for the weights in our list.

So the only weights that could (and actually do) lead to degrees less than or equal to $l^{3}$ are $0, \omega_{l}, \omega_{l-1}$ and $2 \omega_{l}$.

(Case $D_{l}$ ) Here we find the relevant $\lambda$ with very similar arguments as in the cases $B_{l}$ and $C_{l}$.

(Case $A_{l}$ ) Because of the symmetry of the Dynkin diagram, the weights $a_{1} \omega_{1}+$ $\cdots+a_{l} \omega_{l}$ and $a_{l} \omega_{1}+a_{l-1} \omega_{2}+\cdots+a_{1} \omega_{l}$ must describe representations of equal degree (in fact, they are dual to one another). We can again use very similar arguments to those 
Table 2: Small degrees for classical groups of large rank

\begin{tabular}{|c|c|c|c|}
\hline Type & $\lambda$ & $p$ & Degree \\
\hline \multirow[t]{6}{*}{$A_{l}$} & 0 & all & 1 \\
\hline & $\omega_{1}, \omega_{l}$ & all & $l+1$ \\
\hline & $\omega_{2}, \omega_{l-1}$ & all & $l(l+1) / 2$ \\
\hline & $2 \omega_{1}, 2 \omega_{l}$ & all & $(l+1)(l+2) / 2$ \\
\hline & $\omega_{1}+\omega_{l}$ & $p \mid l+1$ & $l^{2}+2 l-1$ \\
\hline & $\omega_{1}+\omega_{l}$ & $p \nmid l+1$ & $l^{2}+2 l$ \\
\hline \multirow[t]{5}{*}{$B_{l}$} & 0 & all & 1 \\
\hline & $\omega_{l}$ & $\neq 2$ & $2 l+1$ \\
\hline & $\omega_{l-1}$ & $\neq 2$ & $2 l^{2}+l$ \\
\hline & $2 \omega_{l}$ & $p \mid 2 l+1$ & $2 l^{2}+3 l-1$ \\
\hline & $2 \omega_{l}$ & $p \nmid 2 l+1$ & $2 l^{2}+3 l$ \\
\hline \multirow[t]{5}{*}{$C_{l}$} & 0 & all & 1 \\
\hline & $\omega_{l}$ & all & $2 l$ \\
\hline & $\omega_{l-1}$ & $p \mid l$ & $2 l^{2}-l-2$ \\
\hline & $\omega_{l-1}$ & $p \nmid l$ & $2 l^{2}-l-1$ \\
\hline & $2 \omega_{l}$ & all & $2 l^{2}+l$ \\
\hline \multirow[t]{6}{*}{$D_{l}$} & 0 & all & 1 \\
\hline & $\omega_{l}$ & all & $2 l$ \\
\hline & $\omega_{l-1}$ & 2 & $2 l^{2}-l-\operatorname{gcd}(2, l)$ \\
\hline & $\omega_{l-1}$ & $\neq 2$ & $2 l^{2}-l$ \\
\hline & $2 \omega_{l}$ & $p \mid l$ & $2 l^{2}+l-2$ \\
\hline & $2 \omega_{l}$ & $p \nmid l$ & $2 l^{2}+l-1$ \\
\hline
\end{tabular}

given above, to find the relevant weights for our list. (Here we rule out $3 \omega_{1}$ and $\omega_{1}+\omega_{2}$ by observing that $3 \omega_{1}-2 \alpha_{1}-\alpha_{2}=\omega_{3}=\omega_{1}+\omega_{2}-\alpha_{1}-\alpha_{2}$.)

It remains to determine the exact degrees of the $L(\lambda)$ in our table. They are probably known to the experts, but we could not find references for all the cases. Type $A_{l}$ and $L\left(\omega_{l}\right)$, and $L\left(\omega_{l-1}\right)$ for the other types, are contained in [13, 5.4.11] and [8, 25.5, Example 8].

We include a proof for the degrees of $L\left(2 \omega_{l}\right)$ and $L\left(\omega_{l-1}\right)$ in the types $B_{l}, C_{l}$ and $D_{l}$, following suggestions given by K. Magaard and G. Hiß.

The idea is to use explicit modules. For all the types, we know the $G$-modules $V\left(X_{l}\right):=L\left(\omega_{l}\right), X \in\{A, B, C, D\}$. These are the natural modules of $\mathrm{SL}_{l+1}, \mathrm{SO}_{2 l+1}, \mathrm{Sp}_{2 l}$ or $\mathrm{SO}_{2 l}$, respectively. We determine the constituents of $V\left(X_{l}\right) \otimes V\left(X_{l}\right)$ for $X \in\{B, C, D\}$. Note that for these types, $V\left(X_{l}\right)$ is self-dual. Since the case $p=2$ is covered by the references above ( $2 \omega_{l}$ is not 2-restricted), we assume in the rest of the proof that $p$ is odd.

We will use the following general remarks. If $V$ is an indecomposable $\overline{\mathbb{F}}_{p} G$-module, then $V \otimes V^{*}$ has the trivial module as direct summand if and only if $p \nmid \operatorname{dim}(V)$. 
(Here, $V^{*}$ denotes the dual module.) In that case, there is exactly one trivial direct summand; see $[1,3.1 .9]$ for a proof. If $v_{1}, \ldots, v_{n}$ is a basis of $V$, then $V \otimes V$ has two submodules $S^{2}(V)$ with basis $\left\{v_{i} \otimes v_{j}+v_{j} \otimes v_{i} \mid 1 \leqslant i<j \leqslant n\right\} \cup\left\{v_{i} \otimes v_{i} \mid 1 \leqslant i \leqslant n\right\}$ and $\Lambda^{2}(V)$ with basis $\left\{v_{i} \otimes v_{j}-v_{j} \otimes v_{i} \mid 1 \leqslant i<j \leqslant n\right\}$. Since $p \neq 2$, we have $V \otimes V=S^{2}(V) \oplus \Lambda^{2}(V)$.

(Case $C_{l}$ ) Let $\left\{v_{i}, v_{i}^{\prime} \mid 1 \leqslant i \leqslant l\right\}$ be a basis of the natural symplectic module $V\left(C_{l}\right)$ such that for the symplectic form $(\cdot, \cdot)$ we have $\left(v_{i}, v_{i}^{\prime}\right)=1=-\left(v_{i}^{\prime}, v_{i}\right)$, where $1 \leqslant i \leqslant l$, and $\left(v_{i}, v_{j}\right)=0=\left(v_{i}, v_{j}^{\prime}\right)$ for all $i \neq j$. Then the vector $\sum_{i=1}^{l}\left(v_{i} \otimes v_{i}^{\prime}-v_{i}^{\prime} \otimes v_{i}\right) \in \Lambda^{2}(V)$ is invariant under the symplectic group. If $p \nmid 2 l$, this vector must span the unique trivial direct summand mentioned above.

The group $G$ contains a subgroup isomorphic to $\mathrm{SL}_{l}\left(\overline{\mathbb{F}}_{p}\right)$. An element acts on the subspace of $V\left(C_{l}\right)$ spanned by $v_{1}, \ldots, v_{l}$ and by its inverse on the subspace spanned by $v_{1}^{\prime}, \ldots v_{l}^{\prime}$. Hence $V\left(C_{l}\right)$ restricted to this subgroup is isomorphic to $V\left(A_{l-1}\right) \oplus V\left(A_{l-1}\right)^{*}$. Restricting the representation $S^{2}\left(V\left(C_{l}\right)\right)$ to this subgroup, we find the decomposition $S^{2}\left(V\left(A_{l-1}\right)\right) \oplus$ $S^{2}\left(V\left(A_{l-1}\right)^{*}\right) \oplus\left(V\left(A_{l-1}\right) \otimes V\left(A_{l-1}\right)^{*}\right)$; see $[4$, I, 12.]. Using the known degrees for case $A_{l-1}$, we see that this contains at most two trivial constituents. The similar restriction to a subgroup of type $C_{l-1}$ shows by induction that one irreducible constituent of $S^{2}\left(V\left(C_{l}\right)\right)$ has degree at least $2(l-1)^{2}+l-1$. Comparing degrees, we see that the three non-trivial constituents in the restriction to $\mathrm{SL}_{l}$ must lie in a single constituent of $S^{2}\left(V\left(C_{l}\right)\right)$. To summarize, we have at most one non-trivial and two trivial constituents. If there are trivial constituents, then one must be in the socle; that is, there must be an invariant vector. This can only be the one that we see in the $\left(V\left(A_{l-1}\right) \otimes V\left(A_{l-1}\right)^{*}\right)$-summand of the restriction to $\mathrm{SL}_{l}$. We can write down such a vector and check that it is not invariant under the whole group $G$; we can apply an element that does not leave the space spanned by $v_{1}, \ldots, v_{l}$ invariant. We have proved that $S^{2}\left(V\left(C_{l}\right)\right)$ is irreducible.

We can argue very similarly for $\Lambda^{2}\left(V\left(C_{l}\right)\right)$. If $p \nmid l$, we find that it is a direct sum of an irreducible and the trivial module found above. If $p \mid l$, we find that there is one non-trivial constituent, exactly one trivial constituent in the socle and at most two trivial constituents. Since in this case the trivial constituent in the socle is not a direct summand, there must be a second trivial constituent in the head, by duality.

If $V\left(C_{l}\right)$ has a highest weight vector $v$, then $v \otimes v$ is contained in $S^{2}\left(V\left(C_{l}\right)\right)$ and has weight $2 \omega_{l}$. Hence $S^{2}\left(V\left(C_{l}\right)\right) \cong L\left(2 \omega_{l}\right)$. The other constituents of the tensor product must correspond to dominant weights smaller than $2 \omega_{l}$; there are only two of them, $2 \omega_{l}-\alpha_{l}=$ $\omega_{l-1}$ and 0 . We see that the non-trivial constituent of $\Lambda^{2}\left(V\left(C_{l}\right)\right)$ is isomorphic to $L\left(\omega_{l-1}\right)$.

(Case $D_{l}$ ) This can be handled by almost exactly the same arguments as the case $C_{l}$. Here the trivial submodule is contained in $S^{2}\left(V\left(D_{l}\right)\right)$.

(Case $B_{l}$ ) In this case we consider the restrictions of $S^{2}\left(V\left(B_{l}\right)\right)$ to subgroups $H_{r}$ of type $B_{r}+D_{l-r}, 0 \leqslant r<l$. This leads to decompositions $S^{2}\left(V\left(B_{r}\right)\right) \oplus S^{2}\left(V\left(D_{l-r}\right)\right) \oplus$ $\left(V\left(D_{l-r}\right) \otimes V\left(B_{r}\right)\right)$. Comparing this decomposition for $r=0$ and $r=l / 2$ or $r=(l-1) / 2$, respectively, and using the results for type $D_{l-r}$ and induction, we see as in type $C_{l}$ that $S^{2}\left(V\left(B_{l}\right)\right)$ has only one non-trivial and maybe a few trivial constituents. There is an $r$ such that $p \nmid(l-r)$ and $p \nmid(2 r+1)$. The decomposition above for this $r$ shows that there are at most two trivial constituents. Furthermore, as in type $D_{l}$, we find a trivial submodule. Either this is a direct summand (if $p \nmid(2 l+1)$ ), and then it is the only trivial constituent, or otherwise there is a second constituent in the head of $S^{2}\left(V\left(B_{l}\right)\right)$. The argument for $\Lambda^{2}\left(V\left(B_{l}\right)\right)$ is again very similar.

This finishes the proof. 
It would be interesting if the degrees in our list could be determined more systematically within the framework of highest-weight modules. Then one could systematically work out generalizations of Theorem 5.1 where the bound $M$ is substituted by any fixed polynomial in $l$.

The following corollary completes the list in [7] which gives all the representations of $G(q)$ in non-defining characteristic of degree at most 250.

Corollary 5.2. For any simple $G$ over $\overline{\mathbb{F}}_{p}$, we find all the p-restricted weights $\lambda$ of $G$ such that $L(\lambda)$ has degree $\leqslant 250$ in the lists given in Theorems 4.4 and 5.1 respectively. Taking Theorem 2.2 into account, this determines all $L(\lambda)$ with degree less than or equal to 250.

Acknowledgements I wish to thank Kay Magaard, Gunter Malle and Gerhard Hiß for useful discussions on the topic of this paper.

\section{References}

1. D. J. Benson, Representations and cohomology I, Cambr. Stud. Adv. Math. 30 (Cambridge University Press, 1991). 144

2. N. Burgoyne, 'Modular representations of some finite groups', Representation theory of finite groups and related topics, Proc. Symp. Pure Math. XXI (Amer. Math. Soc, Providence, RI, 1971) 13-17. 137

3. R. W. CARTER, Simple groups of Lie type (Wiley-Interscience, London, 1972). 138

4. C. W. CURTIS and I. ReINER, Methods of representation theory, vols I and II (Wiley, New York, 1981/1987). 144

5. M. Geck, G. Hiss, F. Lübeck, G. Malle and G. Pfeiffer, 'CHEVIE-A system for computing and processing generic character tables for finite groups of Lie type, Weyl groups and Hecke algebras', Appl. Algebra Engrg. Comm. Comput. 7 (1996) 175-210. 139, 146

6. P. B. Gilkey and G. M. Seitz, 'Some representations of exceptional Lie algebras', Geom. Dedicata 25 (1988) 407-416. 135, 137, 138

7. G. Hiss and G. Malle, 'Low-dimensional representations of quasi-simple groups', LMS J. Comput. Math. 4 (2001) 22-63. 135, 145

8. J. E. Humphreys, Introduction to Lie algebras and representation theory (Springer, New York/Heidelberg/Berlin, 1972). 137, 137, 138, 138, 139, 142, 143

9. J. E. Humphreys, 'Modular representations in finite groups of Lie type', Finite simple groups II (ed. M. J. Collins, Academic Press, London, 1980) 259-290. 136

10. C. JANSEN, K. LuX, R. PARKER and R. WiLsON, An atlas of Brauer characters (The Clarendon Press/Oxford University Press, New York, 1995). 142

11. J. C. JANTZEN, 'Darstellungen halbeinfacher Gruppen und kontravariante Formen', J. Reine Angew. Math. 290 (1977) 117-141. 135

12. J. C. Jantzen, Representations of algebraic groups, Pure Appl. Math. 131 (Academic Press, London, 1987). 136, 138, 139 
13. P. Kleidman and M. LiEBECK, The subgroup structure of the finite classical groups, London Math. Soc. Lecture Note Ser. 129 (Cambridge University Press, 1990). 135, 143

14. F. LÜBECK, 'On the computation of elementary divisors of integer matrices', J. Symbolic Comput., to appear. 139

15. R. V. Moody and J. PAtera, 'Fast recursion formula for weight multiplicities', Bull. Amer. Math. Soc. (N.S.) 7 (1982) 237-242. 139

16. A. A. Premet, 'Weights of infinitesimally irreducible representations of Chevalley groups over a field of prime characteristic', Math. USSR Sb. 61 (1988) 167-183 (translated from the Russian original in Math. Sb. (N.S.)). 139

17. MARTIN SchÖNERT et al., GAP_groups, algorithms and programming, 3rd edn (Lehrstuhl D für Mathematik, Rheinisch Westfälische Technische Hochschule, Aachen, Germany, 1993-1997). 139

18. R. Steinberg, 'Lectures on Chevalley groups' (Yale University, 1967). 147

19. R. Steinberg, 'Endomorphisms of linear algebraic groups', Mem. Amer. Math. Soc. 80 (1968). 137

\section{Appendix A. Tables for groups of small rank}

In this section we give the detailed lists for Theorem 4.4. We start by introducing some notation, and describe the centers of the groups $G$ and the action of the fundamental weights on the center.

\section{Appendix A.1. Ordering of fundamental weights}

For the irreducible types of root systems we choose the following ordering for the simple roots $\alpha_{i}$, the corresponding coroots $\alpha_{i}^{\vee}$ and the fundamental weights $\omega_{i}$, where $1 \leqslant i \leqslant l$. We show the Dynkin diagrams with the node of $\alpha_{i}$ labeled by $i$. (This is the labeling we use in all the databases of the CHEVIE [5] project.)

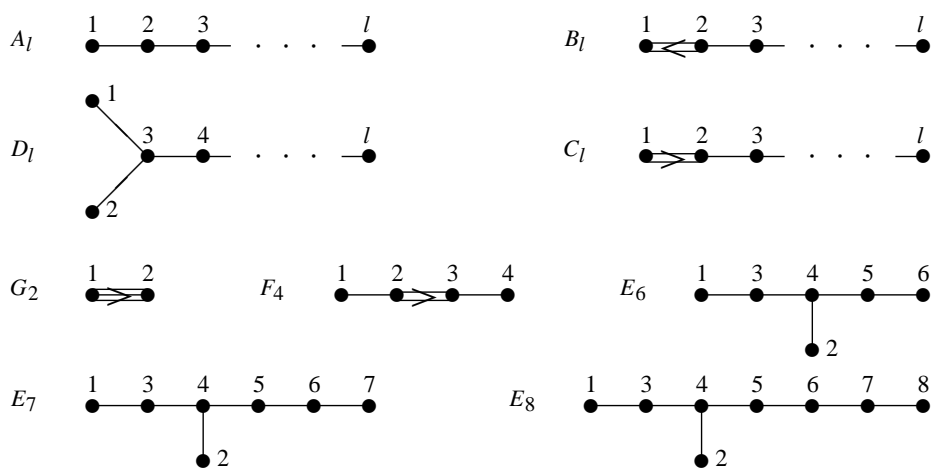


Appendix A.2. Action of fundamental weights on the center of $G$

We give, for each irreducible type of root system, the values of $\omega_{i}(z)$ for $z$ in the center $Z(G)$ of $G$.

To compute this, we use the fact that $Z(G)$ is contained in a maximal torus $T$ of $G$. Such a $T$ is isomorphic to $\left(\sum_{i=1}^{l} \mathbb{Z} \alpha_{i}^{\vee}\right) \otimes_{\mathbb{Z}} \overline{\mathbb{F}}_{p}^{\times}$, and $Z(G)$ consists of those $z \in T$ with $\alpha_{i}(z)=1$ for all $1 \leqslant i \leqslant l$. So we have to solve a system of equations given by the Cartan matrix of the root system.

We denote by $\zeta_{m} \in \overline{\mathbb{F}}_{p}^{\times}$, an element whose multiplicative order is $m$. For $n \in \mathbb{N}$, we write $n_{p^{\prime}}$ for the largest divisor of $n$ which is prime to $p$. For elements $z \in Z(G)$, we write $\underline{\omega}(z):=\left(\omega_{1}(z), \ldots, \omega_{l}(z)\right)$.

( $\left.A_{l}\right) Z(G)$ is cyclic of order $m=(l+1)_{p^{\prime}}$.

It contains a generator $z$ such that $\underline{\omega}(z)=\left(\zeta_{m}, \zeta_{m}^{2}, \ldots, \zeta_{m}^{l}\right)$.

$\left(B_{l}\right) Z(G)$ is cyclic of order $m=\operatorname{gcd}(2, p+1)$.

For the generator $z$ we have $\underline{\omega}(z)=\left(\zeta_{m}, 1, \ldots, 1\right)$.

$\left(C_{l}\right) Z(G)$ is cyclic of order $m=\operatorname{gcd}(2, p+1)$.

For the generator $z$ we have $\underline{\omega}(z)=\left(\zeta_{m}^{l}, \zeta_{m}^{l-1}, \ldots, 1, \zeta_{m}\right)$.

$\left(D_{l}, l\right.$ odd) $Z(G)$ is cyclic of order $m=4$ for odd $p$, and $m=1$ for $p=2$.

There is a generator $z$ such that $\underline{\omega}(z)=\left(\zeta_{m}, \zeta_{m}^{3}, \zeta_{m}^{2}, 1, \zeta_{m}^{2}, 1, \zeta_{m}^{2}, \ldots, 1, \zeta_{m}^{2}\right)$.

$\left(D_{l}, l\right.$ even) $Z(G)$ is elementary abelian of order $m^{2}$,

with $m=2$ if $p$ is odd, and $m=1$ if $p=2$.

There are generators $z_{1}$ and $z_{2}$ such that $\underline{\omega}\left(z_{1}\right)=\left(\zeta_{m}, 1,1, \zeta_{m}, 1, \zeta_{m}, \ldots, 1, \zeta_{m}\right)$

and $\underline{\omega}\left(z_{2}\right)=\left(1, \zeta_{m}, 1, \zeta_{m}, 1, \zeta_{m}, \ldots, 1, \zeta_{m}\right)$.

Here, $z=z_{1} z_{2}$ is the element such that $G /\langle z\rangle \cong \mathrm{SO}_{2 l}\left(\overline{\mathbb{F}}_{p}\right)$.

(E6) $Z(G)$ is cyclic of order $m=3$ if $p \neq 3$, and $m=1$ if $p=3$.

There is a generator $z$ such that $\underline{\omega}(z)=\left(\zeta_{m}, 1, \zeta_{m}^{2}, 1, \zeta_{m}, \zeta_{m}^{2}\right)$.

$\left(E_{7}\right) Z(G)$ is cyclic of order $m=\operatorname{gcd}(2, p+1)$.

For the generator $z$ we have $\underline{\omega}(z)=\left(1, \zeta_{m}, 1,1, \zeta_{m}, 1, \zeta_{m}\right)$.

$\left(G_{2}, F_{4}, E_{8}\right) Z(G)$ is trivial.

\section{Appendix A.3. Frobenius-Schur indicators}

If $p$ is odd, we can determine the Frobenius-Schur indicators of the representations in our lists using a result of Steinberg; see [18, Lemmas 78 and 79].

If $G$ is of type $A_{l}$ or of type $D_{l}$ with odd $l$, or of type $E_{6}$, then the representations $L\left(\sum_{i=1}^{l} a_{i} \omega_{i}\right)$ and $L\left(\sum_{i=1}^{l} a_{\tau(i)} \omega_{i}\right)$, where the permutation $\tau$ is given by the automorphism of order two of the Dynkin diagram, are dual to each other. For other $G$, all $L(\lambda)$ are self-dual.

If $L(\lambda)$ is self-dual (and recall that $p$ is odd) then its Frobenius-Schur indicator is ' + ' if $Z(G)$ has no element of order 2. Otherwise, it is either the sign of $\lambda(z)$ where $z$ is the only element of order 2 in $Z(G)$, or $z=z_{1} z_{2}$ in case $D_{l}$ with even $l$. This can be computed by using Appendix A.2. 


\section{Appendix A.4. An example}

As an example of how to read the data in this appendix, let us determine all the irreducible representations in defining characteristic for groups of type $B_{3}(q) \cong \operatorname{Spin}_{7}(q)$, where $q=p^{f}$, which have degree 448:

We apply Theorems 2.2 and 2.3.

We first need to compute all the factorizations of 448 into factors greater than 1 which appear as degrees in Appendix A.23. Although many of the divisors of 448 appear as degrees, the only such factorizations are $448=7 \cdot 64=7 \cdot 8 \cdot 8$.

Now we take a closer look at Appendix A.23.

If $p=2$, then there is no irreducible representation of degree 7 . Also, the listed representations of degree 448 do not correspond to 2-restricted weights. So, in characteristic 2 there are no irreducible representations of this degree.

For $p \neq 2$, we have representations with $p$-restricted weights of dimension 7 and 8 . If $q \geqslant p^{3}$, there are irreducible representations $L_{i, j, k}$ of degree 448 with highest weights of the form $p^{i} \omega_{3}+p^{j} \omega_{1}+p^{k} \omega_{1}$, where $0 \leqslant i, j, k \leqslant f-1$ and $i, j$ and $k$ are pairwise different.

For $p=3$, there is no irreducible representation of degree 64 , and none with $p$-restricted weight of degree 448. So, there are no further irreducible representations of degree 448 in this case.

If $p=5$, there is $L\left(\omega_{3}\right)$ of degree 7 and $L\left(\omega_{1}+\omega_{2}\right)$ of degree 64 . If $q=5^{f}$, we find for any $0 \leqslant j, k \leqslant f-1$, where $j \neq k$, the irreducible representation

$$
L_{j, k}^{\prime}:=L\left(5^{j}\left(\omega_{1}+\omega_{2}\right)+5^{k} \omega_{3}\right),
$$

restricted to $B_{3}(q)$ of degree 448. Furthermore, we see in Appendix A.23 that $L\left(\omega_{1}+3 \omega_{3}\right)$ and $L\left(2\left(\omega_{1}+\omega_{3}\right)\right)$ have degree 448 .

For larger $p$, there is no representation of degree 64 in our list. We only find $L\left(\omega_{1}+3 \omega_{3}\right)$ if $p \neq 11$, and in the case where $p=7$ also $L\left(3 \omega_{1}+\omega_{2}\right)$.

For all the representations found above, the prime $p$ is odd, and so the center of $G$, and of $G(q)$, is of order 2. Using Appendix A.2, we see that for the non-trivial element $z$ in the center we have $\omega_{1}(z)=-1$ and $\omega_{i}(z)=1$ for $i=2,3$. Applying this to the weights listed above, we find that exactly $L\left(2\left(\omega_{1}+\omega_{3}\right)\right)$ in the case $p=5$ and $L_{i, j, k}$ for $p \neq 2$ are not faithful. From Appendix A.3 we see that these are also the representations with Frobenius-Schur indicator ' + '. The others have indicator ' - '.

\section{Appendix A.5. Reading the tables}

In the tables below, we denote a weight $a_{1} \omega_{1}+\cdots+a_{l} \omega_{l}$ by $\left(a_{1}, \ldots, a_{l}\right)$. When all the values of $a_{i}$ can be written with a single digit, we also suppress the commas.

In type $A_{l}$, the representations

$$
L\left(a_{1} \omega_{1}+\cdots+a_{l} \omega_{l}\right) \quad \text { and } \quad L\left(a_{l} \omega_{1}+a_{l-1} \omega_{2}+\cdots+a_{1} \omega_{l}\right)
$$

are dual to each other; in particular, they have the same dimension. In Appendix A.6 to Appendix A.21 we save some space by including only one of such a pair of representations. 
Appendix A.6. Case $A_{2}, M=400$

(Recall the remark in Appendix A.5.)

\begin{tabular}{|c|c|c|c|c|c|c|c|c|}
\hline deg & $\lambda$ & $p$ & deg & $\lambda$ & $p$ & deg & $\lambda$ & $p$ \\
\hline 1 & (00) & all & 120 & (35) & $\neq 7$ & 255 & (67) & 13 \\
\hline 3 & (01) & all & 123 & (48) & 13 & 260 & (47) & $\neq 11$ \\
\hline 6 & (02) & all & 125 & (44) & $\neq 7$ & 262 & $(6,12)$ & 19 \\
\hline 7 & (11) & 3 & 126 & (57) & 13 & 267 & (48) & 11 \\
\hline 8 & (11) & $\neq 3$ & 127 & (66) & 13 & 267 & $(7,11)$ & 19 \\
\hline 10 & (03) & all & 132 & (27) & all & 270 & $(2,11)$ & $\neq 13$ \\
\hline 15 & (04) & all & 136 & $(0,15)$ & all & 270 & $(8,10)$ & 19 \\
\hline 15 & (12) & all & 143 & $(1,10)$ & all & 271 & (99) & 19 \\
\hline 18 & (13) & 5 & 153 & $(0,16)$ & all & 273 & (56) & $\neq 11$ \\
\hline 19 & (22) & 5 & 154 & (36) & all & 276 & $(0,22)$ & all \\
\hline 21 & (05) & all & 159 & (29) & 11 & 276 & (57) & 11 \\
\hline 24 & (13) & $\neq 5$ & 162 & (28) & $\neq 11$ & 279 & (66) & 11 \\
\hline 27 & (22) & $\neq 5$ & 162 & (45) & 7 & 280 & (39) & $\neq 11,13$ \\
\hline 28 & (06) & all & 165 & (45) & $\neq 7$ & 288 & $(1,15)$ & $\neq 17$ \\
\hline 33 & (15) & 7 & 168 & $(1,11)$ & $\neq 13$ & 297 & $(1,21)$ & 23 \\
\hline 35 & (14) & all & 168 & $(1,15)$ & 17 & 300 & $(0,23)$ & all \\
\hline 36 & (07) & all & 171 & $(0,17)$ & all & 312 & $(2,12)$ & all \\
\hline 36 & (24) & 7 & 171 & (38) & 11 & 315 & (48) & $\neq 11,13$ \\
\hline 37 & (33) & 7 & 179 & (47) & 11 & 316 & $(2,20)$ & 23 \\
\hline 39 & (23) & 5 & 181 & $(2,14)$ & 17 & 323 & $(1,16)$ & all \\
\hline 42 & (23) & $\neq 5$ & 183 & (56) & 11 & 325 & $(0,24)$ & all \\
\hline 45 & (08) & all & 190 & $(0,18)$ & all & 330 & $(3,10)$ & $\neq 13$ \\
\hline 48 & (15) & $\neq 7$ & 192 & (37) & $\neq 11$ & 333 & $(3,19)$ & 23 \\
\hline 55 & (09) & all & 192 & $(3,13)$ & 17 & 336 & (57) & $\neq 11,13$ \\
\hline 60 & (24) & $\neq 7$ & 195 & $(1,12)$ & all & 339 & $(3,11)$ & 13 \\
\hline 63 & (16) & all & 195 & (29) & $\neq 11$ & 343 & (66) & $\neq 11,13$ \\
\hline 63 & (33) & 5 & 201 & $(4,12)$ & 17 & 348 & $(4,18)$ & 23 \\
\hline 64 & (33) & $\neq 5,7$ & 207 & $(1,17)$ & 19 & 351 & $(0,25)$ & all \\
\hline 66 & $(0,10)$ & all & 208 & $(5,11)$ & 17 & 351 & $(2,15)$ & 17 \\
\hline 71 & (25) & 7 & 210 & $(0,19)$ & all & 354 & (49) & 11 \\
\hline 75 & (19) & 11 & 210 & (46) & $\neq 11$ & 357 & $(2,13)$ & all \\
\hline 75 & (34) & 7 & 213 & $(6,10)$ & 17 & 360 & $(1,17)$ & $\neq 19$ \\
\hline 78 & $(0,11)$ & all & 215 & $(2,11)$ & 13 & 360 & $(4,10)$ & 13 \\
\hline 80 & (17) & all & 215 & (55) & 7 & 361 & $(5,17)$ & 23 \\
\hline 81 & (25) & $\neq 7$ & 216 & (55) & $\neq 7,11$ & 370 & (58) & 11 \\
\hline 82 & (28) & 11 & 216 & (79) & 17 & 372 & $(6,16)$ & 23 \\
\hline 87 & (37) & 11 & 217 & (88) & 17 & 375 & $(3,14)$ & 17 \\
\hline 90 & (34) & $\neq 7$ & 222 & $(2,16)$ & 19 & 375 & (49) & $\neq 11,13$ \\
\hline 90 & (46) & 11 & 224 & $(1,13)$ & all & 375 & (59) & 13 \\
\hline 91 & $(0,12)$ & all & 231 & $(0,20)$ & all & 378 & $(0,26)$ & all \\
\hline 91 & (55) & 11 & 231 & $(2,10)$ & $\neq 13$ & 378 & (67) & 11 \\
\hline 99 & (18) & all & 231 & $(3,10)$ & 13 & 381 & $(7,15)$ & 23 \\
\hline 102 & $(1,11)$ & 13 & 234 & (38) & $\neq 11$ & 384 & $(3,11)$ & $\neq 13$ \\
\hline 105 & $(0,13)$ & all & 235 & $(3,15)$ & 19 & 384 & (68) & 13 \\
\hline 105 & (26) & all & 243 & (49) & 13 & 387 & (77) & 13 \\
\hline 111 & $(2,10)$ & 13 & 246 & $(4,14)$ & 19 & 388 & $(8,14)$ & 23 \\
\hline 114 & (35) & 7 & 251 & (58) & 13 & 393 & $(9,13)$ & 23 \\
\hline 117 & (44) & 7 & 252 & (39) & 11 & 395 & $(4,13)$ & 17 \\
\hline 118 & (39) & 13 & 253 & $(0,21)$ & all & 396 & $(10,12)$ & 23 \\
\hline 120 & $(0,14)$ & all & 255 & $(1,14)$ & all & 397 & $(11,11)$ & 23 \\
\hline 120 & (19) & $\neq 11$ & 255 & $(5,13)$ & 19 & 399 & $(1,18)$ & all \\
\hline
\end{tabular}


Appendix A.7. Case $A_{3}, M=500$

(Recall the remark in Appendix A.5.)

\begin{tabular}{rrr||rrr||rrr} 
deg & $\lambda$ & $p$ & deg & $\lambda$ & $p$ & deg & $\lambda$ & $p$ \\
\hline 1 & $(000)$ & all & 120 & $(007)$ & all & 285 & $(303)$ & 7 \\
4 & $(001)$ & all & 120 & $(104)$ & $\neq 7$ & 286 & $(0,0,10)$ & all \\
6 & $(010)$ & all & 124 & $(203)$ & 7 & 294 & $(212)$ & 3 \\
10 & $(002)$ & all & 126 & $(022)$ & $\neq 5$ & 299 & $(222)$ & 5 \\
14 & $(101)$ & 2 & 140 & $(014)$ & all & 300 & $(122)$ & 7 \\
15 & $(101)$ & $\neq 2$ & 140 & $(031)$ & $\neq 5$ & 300 & $(212)$ & $\neq 3,7$ \\
16 & $(011)$ & 3 & 140 & $(112)$ & $\neq 3$ & 300 & $(303)$ & $\neq 7$ \\
19 & $(020)$ & 3 & 149 & $(024)$ & 7 & 315 & $(016)$ & all \\
20 & $(003)$ & all & 156 & $(121)$ & 3 & 334 & $(131)$ & 7 \\
20 & $(011)$ & $\neq 3$ & 160 & $(203)$ & $\neq 7$ & 336 & $(060)$ & $\neq 7$ \\
20 & $(020)$ & $\neq 3$ & 165 & $(008)$ & all & 360 & $(019)$ & 11 \\
32 & $(102)$ & 5 & 173 & $(113)$ & 5 & 360 & $(024)$ & $\neq 7$ \\
35 & $(004)$ & all & 175 & $(121)$ & $\neq 3$ & 360 & $(122)$ & $\neq 5,7$ \\
36 & $(102)$ & $\neq 5$ & 180 & $(033)$ & 7 & 364 & $(0,0,11)$ & all \\
44 & $(111)$ & 3 & 184 & $(131)$ & 5 & 380 & $(115)$ & 7 \\
45 & $(012)$ & all & 189 & $(105)$ & all & 380 & $(213)$ & 5 \\
50 & $(030)$ & all & 192 & $(023)$ & 5 & 384 & $(131)$ & $\neq 5,7$ \\
52 & $(013)$ & 5 & 196 & $(050)$ & all & 396 & $(107)$ & all \\
56 & $(005)$ & all & 206 & $(042)$ & 7 & 416 & $(025)$ & 7 \\
58 & $(111)$ & 5 & 211 & $(113)$ & 7 & 420 & $(108)$ & 11 \\
60 & $(021)$ & all & 216 & $(015)$ & $\neq 7$ & 420 & $(114)$ & all \\
64 & $(111)$ & $\neq 3,5$ & 220 & $(009)$ & all & 420 & $(205)$ & all \\
68 & $(022)$ & 5 & 220 & $(122)$ & 5 & 420 & $(313)$ & 5 \\
69 & $(202)$ & 3 & 224 & $(023)$ & $\neq 5$ & 439 & $(028)$ & 11 \\
70 & $(103)$ & all & 224 & $(051)$ & 7 & 440 & $(017)$ & all \\
80 & $(031)$ & 5 & 231 & $(060)$ & 7 & 455 & $(0,0,12)$ & all \\
83 & $(202)$ & 5 & 235 & $(032)$ & 5 & 460 & $(033)$ & 5 \\
84 & $(006)$ & all & 236 & $(212)$ & 7 & 476 & $(132)$ & 5 \\
84 & $(013)$ & $\neq 5$ & 256 & $(113)$ & $\neq 5,7$ & 480 & $(033)$ & $\neq 5,7$ \\
84 & $(202)$ & $\neq 3,5$ & 260 & $(041)$ & 5 & 480 & $(124)$ & 7 \\
85 & $(040)$ & 5 & 260 & $(204)$ & 7 & 484 & $(151)$ & 7 \\
100 & $(104)$ & 7 & 270 & $(204)$ & $\neq 7$ & 496 & $(304)$ & 7 \\
105 & $(040)$ & $\neq 5$ & 280 & $(032)$ & $\neq 5$ & 500 & $(304)$ & $\neq 7$ \\
116 & $(015)$ & 7 & 280 & $(041)$ & $\neq 5$ & & & \\
116 & $(112)$ & 3 & 280 & $(106)$ & all & & & \\
& & & & & & &
\end{tabular}


Appendix A.8. Case $A_{4}, M=1000$

(Recall the remark in Appendix A.5.)

\begin{tabular}{rrr||rrr||rrr} 
deg & $\lambda$ & $p$ & deg & $\lambda$ & $p$ & deg & $\lambda$ & $p$ \\
\hline 1 & $(0000)$ & all & 185 & $(0022)$ & 5 & 510 & $(0112)$ & 3 \\
5 & $(0001)$ & all & 195 & $(0201)$ & 3 & 535 & $(1013)$ & 5 \\
10 & $(0010)$ & all & 199 & $(2002)$ & 3 & 540 & $(0104)$ & $\neq 7$ \\
15 & $(0002)$ & all & 200 & $(2002)$ & $\neq 3,7$ & 545 & $(0202)$ & 5 \\
23 & $(1001)$ & 5 & 210 & $(0006)$ & all & 560 & $(0031)$ & $\neq 5$ \\
24 & $(1001)$ & $\neq 5$ & 210 & $(0201)$ & $\neq 3,5$ & 560 & $(0202)$ & $\neq 3,5$ \\
30 & $(0011)$ & 3 & 224 & $(0013)$ & $\neq 5$ & 560 & $(1005)$ & all \\
35 & $(0003)$ & all & 235 & $(0111)$ & 5 & 615 & $(1021)$ & 5 \\
40 & $(0011)$ & $\neq 3$ & 255 & $(0031)$ & 5 & 640 & $(0033)$ & 7 \\
40 & $(0101)$ & 2 & 280 & $(0103)$ & all & 670 & $(0023)$ & 5 \\
45 & $(0020)$ & 3 & 280 & $(0111)$ & $\neq 3,5$ & 670 & $(1021)$ & 7 \\
45 & $(0101)$ & $\neq 2$ & 305 & $(0120)$ & 5 & 683 & $(1022)$ & 5 \\
50 & $(0020)$ & $\neq 3$ & 315 & $(0120)$ & $\neq 5$ & 700 & $(0112)$ & $\neq 3$ \\
51 & $(0110)$ & 3 & 315 & $(1004)$ & all & 700 & $(0301)$ & all \\
65 & $(1002)$ & 3 & 320 & $(0040)$ & 5 & 715 & $(0009)$ & all \\
70 & $(0004)$ & all & 325 & $(0015)$ & 7 & 720 & $(0015)$ & $\neq 7$ \\
70 & $(1002)$ & $\neq 3$ & 330 & $(0007)$ & all & 720 & $(1021)$ & $\neq 5,7$ \\
74 & $(0110)$ & 2 & 365 & $(0130)$ & 5 & 765 & $(0203)$ & 7 \\
75 & $(0110)$ & $\neq 2,3$ & 375 & $(1102)$ & 3 & 794 & $(1111)$ & 5 \\
103 & $(0102)$ & 5 & 381 & $(0220)$ & 5 & 826 & $(0042)$ & 7 \\
105 & $(0012)$ & all & 390 & $(0202)$ & 3 & 835 & $(0211)$ & 3 \\
121 & $(0013)$ & 5 & 395 & $(0104)$ & 7 & 840 & $(0023)$ & $\neq 5$ \\
126 & $(0005)$ & all & 410 & $(1012)$ & 7 & 855 & $(0121)$ & 3 \\
126 & $(0102)$ & $\neq 5$ & 410 & $(1102)$ & 5 & 875 & $(2004)$ & all \\
135 & $(1011)$ & 3 & 420 & $(0014)$ & all & 895 & $(0113)$ & 5 \\
145 & $(1003)$ & 7 & 420 & $(0022)$ & $\neq 5$ & 924 & $(1006)$ & all \\
160 & $(1003)$ & $\neq 7$ & 435 & $(1102)$ & 7 & 945 & $(0105)$ & all \\
160 & $(1011)$ & 2 & 445 & $(2003)$ & 7 & 945 & $(1013)$ & $\neq 5$ \\
165 & $(0111)$ & 3 & 450 & $(1012)$ & $\neq 7$ & 949 & $(1111)$ & 7 \\
170 & $(0201)$ & 5 & 450 & $(2003)$ & $\neq 7$ & 955 & $(0032)$ & 5 \\
174 & $(0021)$ & 3 & 470 & $(0024)$ & 7 & 980 & $(0130)$ & $\neq 5$ \\
175 & $(0021)$ & $\neq 3$ & 476 & $(1111)$ & 3 & 999 & $(3003)$ & 7 \\
175 & $(0030)$ & all & 480 & $(1102)$ & $\neq 3,5,7$ & 1000 & $(3003)$ & $\neq 7$ \\
175 & $(1011)$ & $\neq 2,3$ & 490 & $(0040)$ & $\neq 5$ & & & \\
176 & $(2002)$ & 7 & 495 & $(0008)$ & all & & &
\end{tabular}


Appendix A.9. Case $A_{5}, M=2500$

(Recall the remark in Appendix A.5.)

\begin{tabular}{|c|c|c|c|c|c|c|c|c|}
\hline deg & $\lambda$ & $p$ & deg & $\lambda$ & $p$ & deg & $\lambda$ & $p$ \\
\hline 1 & $(00000)$ & all & 426 & $(00022)$ & 5 & 1246 & (00130) & 5 \\
\hline 6 & (00001) & all & 440 & (02001) & 3 & 1251 & (00024) & 7 \\
\hline 15 & (00010) & all & 462 & (00006) & all & 1260 & (11002) & $\neq 3$ \\
\hline 20 & (00100) & all & 474 & (00111) & 3 & 1287 & (00008) & all \\
\hline 21 & (00002) & all & 490 & $(00030)$ & all & 1338 & (10102) & 5 \\
\hline 34 & (10001) & 2 & 504 & (00013) & $\neq 5$ & 1365 & (00210) & 5 \\
\hline 34 & (10001) & 3 & 520 & (10101) & 7 & 1386 & (10005) & all \\
\hline 35 & (10001) & $\neq 2,3$ & 540 & (10101) & $\neq 2,7$ & 1420 & (01110) & 5 \\
\hline 50 & (00011) & 3 & 560 & (02001) & $\neq 3$ & 1431 & (02002) & 7 \\
\hline 56 & (00003) & all & 606 & (01003) & 7 & 1449 & (00210) & 3 \\
\hline 70 & (00011) & $\neq 3$ & 630 & (00201) & 5 & 1470 & (00210) & $\neq 3,5$ \\
\hline 78 & (01001) & 5 & 630 & (01011) & 3 & 1506 & (00220) & 5 \\
\hline 84 & (01001) & $\neq 5$ & 666 & $(00031)$ & 5 & 1539 & (00202) & 3 \\
\hline 90 & (00020) & 3 & 666 & (01101) & 3 & 1569 & (02002) & 3 \\
\hline 90 & (00101) & 2 & 700 & (10004) & all & 1575 & (01004) & all \\
\hline 105 & (00020) & $\neq 3$ & 708 & (00111) & 5 & 1674 & (00112) & 3 \\
\hline 105 & (00101) & $\neq 2$ & 720 & (00201) & 3 & 1686 & (00310) & 5 \\
\hline 114 & (10002) & 7 & 720 & (01003) & $\neq 7$ & 1701 & (10102) & $\neq 5$ \\
\hline 120 & (10002) & $\neq 7$ & 720 & (01011) & 2 & 1751 & (00400) & 5 \\
\hline 126 & (00004) & all & 786 & (00015) & 7 & 1764 & (00031) & $\neq 5$ \\
\hline 126 & (00110) & 3 & 792 & (00007) & all & 1764 & (00040) & $\neq 5$ \\
\hline 141 & (00200) & 3 & 804 & (11002) & 3 & 1800 & (00104) & $\neq 7$ \\
\hline 154 & (01010) & 2 & 813 & (01020) & 5 & 1800 & (02002) & $\neq 3,7$ \\
\hline 175 & (00200) & $\neq 3$ & 840 & (00103) & all & 1876 & (00033) & 7 \\
\hline 188 & (01010) & 5 & 840 & (00201) & $\neq 3,5$ & 1890 & (00023) & 5 \\
\hline 189 & (01010) & $\neq 2,5$ & 840 & (01011) & $\neq 2,3$ & 1960 & (01110) & $\neq 3,5$ \\
\hline 204 & (00110) & 2 & 896 & (00111) & $\neq 3,5$ & 1974 & (10021) & 3 \\
\hline 210 & (00012) & all & 924 & (01101) & 2 & 1980 & (00015) & $\neq 7$ \\
\hline 210 & (00110) & $\neq 2,3$ & 951 & $(00040)$ & 5 & 1995 & (20004) & 5 \\
\hline 246 & (00013) & 5 & 960 & (00300) & 5 & 2002 & (00009) & all \\
\hline 246 & (01002) & 3 & 960 & (01110) & 3 & 2024 & (11011) & 3 \\
\hline 252 & (00005) & all & 980 & $(00300)$ & $\neq 5$ & 2061 & (01012) & 7 \\
\hline 258 & (00102) & 5 & 1050 & (00014) & all & 2100 & (03001) & 7 \\
\hline 279 & (10011) & 3 & 1050 & (01101) & $\neq 2,3$ & 2106 & (01102) & 3 \\
\hline 280 & (01002) & $\neq 3$ & 1050 & (20003) & all & 2205 & (10021) & $\neq 3$ \\
\hline 315 & (10003) & all & 1071 & (01020) & 3 & 2268 & (10006) & 11 \\
\hline 336 & (00102) & $\neq 5$ & 1078 & (10012) & 5 & 2290 & (30003) & 5 \\
\hline 363 & (10011) & 5 & 1098 & (00120) & 5 & 2310 & (10022) & 5 \\
\hline 369 & (10011) & 7 & 1134 & (00022) & $\neq 5$ & 2310 & (20004) & $\neq 5$ \\
\hline 384 & (10011) & $\neq 3,5,7$ & 1134 & (10012) & $\neq 5$ & 2364 & (10201) & 5 \\
\hline 400 & (10101) & 2 & 1170 & $(00120)$ & 3 & 2415 & (00202) & 5 \\
\hline 404 & (20002) & 7 & 1176 & (00120) & $\neq 3,5$ & 2430 & (01012) & $\neq 7$ \\
\hline 405 & (20002) & $\neq 7$ & 1176 & (01020) & $\neq 3,5$ & 2430 & (10111) & 3 \\
\hline 414 & (00021) & 3 & 1194 & (00104) & 7 & & & \\
\hline 420 & $(00021)$ & $\neq 3$ & 1224 & (10013) & 5 & & & \\
\hline
\end{tabular}


Small degree representations in defining characteristic

Appendix A.10. Case $A_{6}, M=2800$

(Recall the remark in Appendix A.5.)

\begin{tabular}{rrr||rrr||rrr} 
deg & $\lambda$ & $p$ & deg & $\lambda$ & $p$ & deg & $\lambda$ & $p$ \\
\hline 1 & $(000000)$ & all & 490 & $(000110)$ & $\neq 2,3$ & 1428 & $(010011)$ & 3 \\
7 & $(000001)$ & all & 490 & $(000200)$ & $\neq 3$ & 1520 & $(000031)$ & 5 \\
21 & $(000010)$ & all & 493 & $(010002)$ & 7 & 1575 & $(010003)$ & all \\
28 & $(000002)$ & all & 540 & $(010002)$ & $\neq 7$ & 1701 & $(011001)$ & 3 \\
35 & $(000100)$ & all & 553 & $(000102)$ & 5 & 1709 & $(000015)$ & 7 \\
47 & $(100001)$ & 7 & 560 & $(100003)$ & all & 1716 & $(000007)$ & all \\
48 & $(100001)$ & $\neq 7$ & 581 & $(001010)$ & 5 & 1771 & $(000111)$ & 5 \\
77 & $(000011)$ & 3 & 588 & $(001010)$ & $\neq 2,5$ & 1827 & $(010011)$ & 5 \\
84 & $(000003)$ & all & 687 & $(200002)$ & 3 & 1863 & $(000201)$ & 5 \\
112 & $(000011)$ & $\neq 3$ & 707 & $(001002)$ & 3 & 1876 & $(010011)$ & 7 \\
133 & $(010001)$ & 2 & 707 & $(100011)$ & 2 & 1907 & $(001003)$ & 7 \\
133 & $(010001)$ & 3 & 735 & $(100011)$ & $\neq 2,3$ & 1953 & $(110002)$ & 3 \\
140 & $(010001)$ & $\neq 2,3$ & 735 & $(200002)$ & $\neq 3$ & 1967 & $(002001)$ & 3 \\
161 & $(000020)$ & 3 & 736 & $(001100)$ & 2 & 1967 & $(200003)$ & 5 \\
175 & $(000101)$ & 2 & 756 & $(000102)$ & $\neq 5$ & 2016 & $(010011)$ & $\neq 3,5,7$ \\
189 & $(100002)$ & all & 783 & $(001100)$ & 5 & 2100 & $(000103)$ & all \\
196 & $(000020)$ & $\neq 3$ & 784 & $(001100)$ & $\neq 2,3,5$ & 2106 & $(000201)$ & 3 \\
203 & $(001001)$ & 5 & 840 & $(001002)$ & $\neq 3$ & 2155 & $(001011)$ & 3 \\
210 & $(000004)$ & all & 861 & $(000021)$ & 3 & 2156 & $(200003)$ & $\neq 5$ \\
210 & $(000101)$ & $\neq 2$ & 875 & $(000022)$ & 5 & 2198 & $(010101)$ & 2 \\
224 & $(001001)$ & $\neq 5$ & 882 & $(000021)$ & $\neq 3$ & 2310 & $(000014)$ & all \\
266 & $(000110)$ & 3 & 924 & $(000006)$ & all & 2331 & $(100012)$ & 3 \\
344 & $(010010)$ & 5 & 1008 & $(000013)$ & $\neq 5$ & 2352 & $(000111)$ & $\neq 3,5$ \\
357 & $(000200)$ & 3 & 1050 & $(020001)$ & 3 & 2387 & $(010020)$ & 3 \\
378 & $(000012)$ & all & 1113 & $(100101)$ & 2 & 2394 & $(001101)$ & 3 \\
391 & $(010010)$ & 3 & 1148 & $(000111)$ & 3 & 2400 & $(001003)$ & $\neq 7$ \\
392 & $(010010)$ & $\neq 3,5$ & 1148 & $(020001)$ & 7 & 2400 & $(001011)$ & 2 \\
393 & $(001100)$ & 3 & 1176 & $(000030)$ & all & 2415 & $(000040)$ & 5 \\
448 & $(001010)$ & 2 & 1211 & $(100101)$ & 5 & 2450 & $(100012)$ & $\neq 3$ \\
455 & $(000013)$ & 5 & 1260 & $(020001)$ & $\neq 3,7$ & 2611 & $(110002)$ & 7 \\
462 & $(000005)$ & all & 1302 & $(100004)$ & 5 & 2646 & $(000022)$ & $\neq 5$ \\
469 & $(000110)$ & 2 & 1323 & $(100101)$ & $\neq 2,5$ & 2646 & $(000201)$ & $\neq 3,5$ \\
483 & $(100011)$ & 3 & 1386 & $(100004)$ & $\neq 5$ & 2800 & $(110002)$ & $\neq 3,7$
\end{tabular}


Appendix A.11. Case $A_{7}, M=3000$

(Recall the remark in Appendix A.5.)

\begin{tabular}{rrr||rrr||rrr} 
deg & $\lambda$ & $p$ & deg & $\lambda$ & $p$ & deg & $\lambda$ & $p$ \\
\hline 1 & $(0000000)$ & all & 658 & $(0100010)$ & 2 & 1592 & $(0010002)$ & 7 \\
8 & $(0000001)$ & all & 719 & $(0100010)$ & 7 & 1624 & $(0000021)$ & 3 \\
28 & $(0000010)$ & all & 720 & $(0100010)$ & $\neq 2,3,7$ & 1632 & $(0010100)$ & 2 \\
36 & $(0000002)$ & all & 784 & $(0000013)$ & 5 & 1652 & $(0000022)$ & 5 \\
56 & $(0000100)$ & all & 784 & $(0000200)$ & 3 & 1680 & $(0000021)$ & $\neq 3$ \\
62 & $(1000001)$ & 2 & 792 & $(0000005)$ & all & 1708 & $(0001002)$ & 3 \\
63 & $(1000001)$ & $\neq 2$ & 860 & $(1000011)$ & 3 & 1716 & $(0000006)$ & all \\
70 & $(0001000)$ & all & 888 & $(1000003)$ & 5 & 1763 & $(0002000)$ & 5 \\
112 & $(0000011)$ & 3 & 924 & $(1000003)$ & $\neq 5$ & 1764 & $(0002000)$ & $\neq 3,5$ \\
120 & $(0000003)$ & all & 945 & $(0100002)$ & all & 1800 & $(0010002)$ & $\neq 7$ \\
168 & $(0000011)$ & $\neq 3$ & 952 & $(0000110)$ & 2 & 1848 & $(0000013)$ & $\neq 5$ \\
208 & $(0100001)$ & 7 & 1008 & $(0000110)$ & $\neq 2,3$ & 2016 & $(0200001)$ & 3 \\
216 & $(0100001)$ & $\neq 7$ & 1016 & $(0001100)$ & 3 & 2100 & $(0001002)$ & $\neq 3$ \\
266 & $(0000020)$ & 3 & 1064 & $(0000102)$ & 5 & 2128 & $(1000101)$ & 2 \\
272 & $(1000002)$ & 3 & 1092 & $(0001010)$ & 2 & 2136 & $(0001100)$ & 2 \\
280 & $(1000002)$ & $\neq 3$ & 1107 & $(0002000)$ & 3 & 2289 & $(0010100)$ & 5 \\
308 & $(0000101)$ & 2 & 1128 & $(0010010)$ & 5 & 2344 & $(0001100)$ & 5 \\
330 & $(0000004)$ & all & 1169 & $(2000002)$ & 5 & 2352 & $(0001100)$ & $\neq 2,3,5$ \\
336 & $(0000020)$ & $\neq 3$ & 1176 & $(0000200)$ & $\neq 3$ & 2352 & $(0010100)$ & $\neq 2,5$ \\
378 & $(0000101)$ & $\neq 2$ & 1231 & $(2000002)$ & 3 & 2400 & $(1000004)$ & 11 \\
392 & $(0010001)$ & 2 & 1232 & $(2000002)$ & $\neq 3,5$ & 2464 & $(0000111)$ & 3 \\
392 & $(0010001)$ & 3 & 1244 & $(1000011)$ & 7 & 2520 & $(0000030)$ & all \\
420 & $(0010001)$ & $\neq 2,3$ & 1280 & $(1000011)$ & $\neq 3,7$ & 2520 & $(0200001)$ & $\neq 3$ \\
448 & $(0001001)$ & 5 & 1336 & $(0010010)$ & 3 & 2520 & $(1000004)$ & $\neq 11$ \\
504 & $(0000110)$ & 3 & 1344 & $(0010010)$ & $\neq 3,5$ & 2584 & $(0100011)$ & 3 \\
504 & $(0001001)$ & $\neq 5$ & 1484 & $(0001010)$ & 5 & 2632 & $(1000101)$ & 3 \\
630 & $(0000012)$ & all & 1512 & $(0000102)$ & $\neq 5$ & 2800 & $(1000101)$ & $\neq 2,3$ \\
657 & $(0100010)$ & 3 & 1512 & $(0001010)$ & $\neq 2,5$ & 2828 & $(1001001)$ & 5
\end{tabular}

Appendix A.12. Case $A_{8}, M=4000$

(Recall the remark in Appendix A.5.)

\begin{tabular}{rrr||rrr||rrr} 
deg & $\lambda$ & $p$ & deg & $\lambda$ & $p$ & deg & $\lambda$ & $p$ \\
\hline 1 & $(00000000)$ & all & 966 & $(00010001)$ & 2 & 2079 & $(10000011)$ & $\neq 2,3,5$ \\
9 & $(00000001)$ & all & 966 & $(00010001)$ & 3 & 2304 & $(00001100)$ & 3 \\
36 & $(00000010)$ & all & 990 & $(00000012)$ & all & 2352 & $(00001010)$ & 2 \\
45 & $(00000002)$ & all & 1008 & $(00001001)$ & $\neq 5$ & 2385 & $(00100010)$ & 3 \\
79 & $(10000001)$ & 3 & 1050 & $(00010001)$ & $\neq 2,3$ & 2394 & $(00100010)$ & 2 \\
80 & $(10000001)$ & $\neq 3$ & 1135 & $(01000010)$ & 7 & 2520 & $(00000200)$ & $\neq 3$ \\
84 & $(00000100)$ & all & 1214 & $(01000010)$ & 2 & 2691 & $(00100010)$ & 7 \\
126 & $(00001000)$ & all & 1215 & $(01000010)$ & $\neq 2,7$ & 2700 & $(00100010)$ & $\neq 2,3,7$ \\
156 & $(00000011)$ & 3 & 1278 & $(00000013)$ & 5 & 2772 & $(00000102)$ & $\neq 5$ \\
165 & $(00000003)$ & all & 1287 & $(00000005)$ & all & 2844 & $(00000021)$ & 3 \\
240 & $(00000011)$ & $\neq 3$ & 1359 & $(10000011)$ & 3 & 2907 & $(00002000)$ & 3 \\
306 & $(01000001)$ & 2 & 1395 & $(10000003)$ & 11 & 2922 & $(00000022)$ & 5 \\
315 & $(01000001)$ & $\neq 2$ & 1440 & $(10000003)$ & $\neq 11$ & 2970 & $(00000021)$ & $\neq 3$ \\
387 & $(10000002)$ & 5 & 1461 & $(01000002)$ & 3 & 3003 & $(00000006)$ & all \\
396 & $(10000002)$ & $\neq 5$ & 1540 & $(01000002)$ & $\neq 3$ & 3060 & $(00010010)$ & 5 \\
414 & $(00000020)$ & 3 & 1554 & $(00000200)$ & 3 & 3139 & $(00011000)$ & 3 \\
495 & $(00000004)$ & all & 1764 & $(00000110)$ & 2 & 3168 & $(00000013)$ & $\neq 5$ \\
504 & $(00000101)$ & 2 & 1864 & $(20000002)$ & 11 & 3318 & $(00001010)$ & 5 \\
540 & $(00000020)$ & $\neq 3$ & 1890 & $(00000102)$ & 5 & 3402 & $(00001010)$ & $\neq 2,5$ \\
630 & $(00000101)$ & $\neq 2$ & 1890 & $(00000110)$ & $\neq 2,3$ & 3414 & $(02000001)$ & 3 \\
684 & $(00100001)$ & 7 & 1943 & $(20000002)$ & 5 & 3465 & $(00100002)$ & all \\
720 & $(00100001)$ & $\neq 7$ & 1944 & $(20000002)$ & $\neq 5,11$ & 3654 & $(00001002)$ & 3 \\
882 & $(00000110)$ & 3 & 2034 & $(10000011)$ & 2 & 3744 & $(00010010)$ & 3 \\
882 & $(00001001)$ & 5 & 2043 & $(10000011)$ & 5 & 3780 & $(00010010)$ & $\neq 3,5$
\end{tabular}


Appendix A.13. Case A9, $M=6000$

(Recall the remark in Appendix A.5.)

\begin{tabular}{rrr||rrr||rrr} 
deg & \multicolumn{1}{c}{$\lambda$} & $p$ & deg & $\lambda$ & $p$ & deg & $\lambda$ & $p$ \\
\hline 1 & $(000000000)$ & all & 1110 & $(001000001)$ & 2 & 2924 & $(200000002)$ & 11 \\
10 & $(000000001)$ & all & 1155 & $(001000001)$ & $\neq 2$ & 2925 & $(200000002)$ & $\neq 3,11$ \\
45 & $(000000010)$ & all & 1452 & $(000000110)$ & 3 & 3048 & $(000000110)$ & 2 \\
55 & $(000000002)$ & all & 1485 & $(000000012)$ & all & 3155 & $(100000011)$ & 11 \\
98 & $(100000001)$ & 2 & 1596 & $(000001001)$ & 5 & 3156 & $(000000102)$ & 5 \\
98 & $(100000001)$ & 5 & 1826 & $(010000010)$ & 2 & 3200 & $(100000011)$ & $\neq 3,11$ \\
99 & $(100000001)$ & $\neq 2,5$ & 1848 & $(000001001)$ & $\neq 5$ & 3300 & $(000000110)$ & $\neq 2,3$ \\
120 & $(000000100)$ & all & 1860 & $(000100001)$ & 7 & 4510 & $(001000010)$ & 7 \\
210 & $(000000011)$ & 3 & 1924 & $(010000010)$ & 3 & 4620 & $(000001010)$ & 2 \\
210 & $(000001000)$ & all & 1925 & $(010000010)$ & $\neq 2,3$ & 4698 & $(000000021)$ & 3 \\
220 & $(000000003)$ & all & 1980 & $(000100001)$ & $\neq 7$ & 4740 & $(000001100)$ & 3 \\
252 & $(000010000)$ & all & 1990 & $(100000011)$ & 3 & 4752 & $(000000102)$ & $\neq 5$ \\
330 & $(000000011)$ & $\neq 3$ & 1992 & $(000000013)$ & 5 & 4905 & $(000000022)$ & 5 \\
430 & $(010000001)$ & 3 & 2002 & $(000000005)$ & all & 4940 & $(001000010)$ & 2 \\
440 & $(010000001)$ & $\neq 3$ & 2100 & $(000010001)$ & 2 & 4950 & $(000000021)$ & $\neq 3$ \\
530 & $(100000002)$ & 11 & 2100 & $(000010001)$ & 3 & 4950 & $(000000200)$ & $\neq 3$ \\
540 & $(100000002)$ & $\neq 11$ & 2145 & $(100000003)$ & all & 4950 & $(001000010)$ & $\neq 2,7$ \\
615 & $(000000020)$ & 3 & 2278 & $(010000002)$ & 5 & 5005 & $(000000006)$ & all \\
715 & $(000000004)$ & all & 2310 & $(000010001)$ & $\neq 2,3$ & 5148 & $(000000013)$ & $\neq 5$ \\
780 & $(000000101)$ & 2 & 2376 & $(010000002)$ & $\neq 5$ & 5730 & $(001000002)$ & 3 \\
825 & $(000000020)$ & $\neq 3$ & 2826 & $(200000002)$ & 3 & 5940 & $(020000001)$ & 3 \\
990 & $(000000101)$ & $\neq 2$ & 2850 & $(000000200)$ & 3 & & &
\end{tabular}

Appendix A.14. Case $A_{10}, M=10000$

(Recall the remark in Appendix A.5.)

\begin{tabular}{rrr||rrr||rrr} 
deg & $\lambda$ & $p$ & deg & $\lambda$ & $p$ & deg & $\lambda$ & $p$ \\
\hline 1 & $(0000000000)$ & all & 1760 & $(0010000001)$ & $\neq 3$ & 4620 & $(0000010001)$ & $\neq 2,3$ \\
11 & $(0000000001)$ & all & 2145 & $(0000000012)$ & all & 4653 & $(1000000011)$ & 2 \\
55 & $(0000000010)$ & all & 2277 & $(0000000110)$ & 3 & 4653 & $(1000000011)$ & 5 \\
66 & $(0000000002)$ & all & 2706 & $(0000001001)$ & 5 & 4719 & $(1000000011)$ & $\neq 2,3,5$ \\
119 & $(1000000001)$ & 11 & 2784 & $(0100000010)$ & 3 & 4752 & $(0000100001)$ & $\neq 7$ \\
120 & $(1000000001)$ & $\neq 11$ & 2903 & $(0100000010)$ & 5 & 4917 & $(0000000200)$ & 3 \\
165 & $(0000000100)$ & all & 2904 & $(0100000010)$ & $\neq 3,5$ & 4983 & $(0000000110)$ & 2 \\
275 & $(0000000011)$ & 3 & 2959 & $(1000000011)$ & 3 & 5016 & $(0000000102)$ & 5 \\
286 & $(0000000003)$ & all & 2992 & $(0000000013)$ & 5 & 5445 & $(0000000110)$ & $\neq 2,3$ \\
330 & $(0000001000)$ & all & 3003 & $(0000000005)$ & all & 7403 & $(0000000021)$ & 3 \\
440 & $(0000000011)$ & $\neq 3$ & 3014 & $(1000000003)$ & 13 & 7722 & $(0000000102)$ & $\neq 5$ \\
462 & $(0000010000)$ & all & 3080 & $(1000000003)$ & $\neq 13$ & 7865 & $(0000000021)$ & $\neq 3$ \\
583 & $(0100000001)$ & 2 & 3168 & $(0000001001)$ & $\neq 5$ & 7876 & $(0010000010)$ & 2 \\
583 & $(0100000001)$ & 5 & 3300 & $(0001000001)$ & 2 & 7887 & $(0000000022)$ & 5 \\
594 & $(0100000001)$ & $\neq 2,5$ & 3391 & $(0100000002)$ & 11 & 8008 & $(0000000006)$ & all \\
704 & $(1000000002)$ & 3 & 3465 & $(0001000001)$ & $\neq 2$ & 8008 & $(0000000013)$ & $\neq 5$ \\
715 & $(1000000002)$ & $\neq 3$ & 3510 & $(0100000002)$ & $\neq 11$ & 8448 & $(0000001010)$ & 2 \\
880 & $(0000000020)$ & 3 & 4115 & $(2000000002)$ & 13 & 8459 & $(0010000010)$ & 3 \\
1001 & $(0000000004)$ & all & 4158 & $(0000010001)$ & 2 & 8470 & $(0010000010)$ & $\neq 2,3$ \\
1155 & $(0000000101)$ & 2 & 4158 & $(0000010001)$ & 3 & 9042 & $(0000001100)$ & 3 \\
1210 & $(0000000020)$ & $\neq 3$ & 4234 & $(2000000002)$ & 3 & 9075 & $(0000000200)$ & $\neq 3$ \\
1485 & $(0000000101)$ & $\neq 2$ & 4235 & $(2000000002)$ & $\neq 3,13$ & 9405 & $(0200000001)$ & 3 \\
1705 & $(0010000001)$ & 3 & 4422 & $(0000100001)$ & 7 & 9713 & $(0010000002)$ & 5
\end{tabular}


Appendix A.15. Case $A_{11}, M=12000$

(Recall the remark in Appendix A.5.)

\begin{tabular}{|c|c|c|c|c|c|c|c|c|}
\hline deg & $\lambda$ & $p$ & deg & $\lambda$ & $p$ & deg & $\lambda$ & $p$ \\
\hline 1 & $(00000000000)$ & all & 1716 & $(00000000020)$ & $\neq 3$ & 5720 & $(00010000001)$ & $\neq 3$ \\
\hline 12 & $(00000000001)$ & all & 2145 & $(00000000101)$ & $\neq 2$ & 5797 & $(20000000002)$ & 7 \\
\hline 66 & (00000000010) & all & 2508 & (00100000001) & 2 & 5939 & (20000000002) & 13 \\
\hline 78 & (00000000002) & all & 2508 & (00100000001) & 5 & 5940 & (20000000002) & $\neq 7,13$ \\
\hline 142 & (10000000001) & 2 & 2574 & (00100000001) & $\neq 2,5$ & 6642 & (10000000011) & 11 \\
\hline 142 & (10000000001) & 3 & 3003 & $(00000000012)$ & all & 6654 & $(10000000011)$ & 13 \\
\hline 143 & $(10000000001)$ & $\neq 2,3$ & 3432 & $(00000000110)$ & 3 & 6720 & (10000000011) & $\neq 3,11,13$ \\
\hline 220 & (00000000100) & all & 4069 & $(01000000010)$ & 5 & 7656 & (00000000102) & 5 \\
\hline 352 & (00000000011) & 3 & 4070 & $(01000000010)$ & 2 & 7656 & (00000010001) & 2 \\
\hline 364 & (00000000003) & all & 4146 & (10000000011) & 3 & 7656 & (00000010001) & 3 \\
\hline 495 & $(00000001000)$ & all & 4211 & $(01000000010)$ & 11 & 7788 & $(00000000110)$ & 2 \\
\hline 572 & $(00000000011)$ & $\neq 3$ & 4212 & $(01000000010)$ & $\neq 2,5,11$ & 8074 & $(00000000200)$ & 3 \\
\hline 768 & (01000000001) & 11 & 4212 & (10000000003) & 7 & 8514 & $(00001000001)$ & 2 \\
\hline 780 & (01000000001) & $\neq 11$ & 4290 & (10000000003) & $\neq 7$ & 8580 & (00000000110) & $\neq 2,3$ \\
\hline 792 & $(00000010000)$ & all & 4356 & $(00000000013)$ & 5 & 8580 & (00000010001) & $\neq 2,3$ \\
\hline 912 & (10000000002) & 13 & 4356 & $(00000001001)$ & 5 & 9009 & $(00001000001)$ & $\neq 2$ \\
\hline 924 & $(00000100000)$ & all & 4368 & (00000000005) & all & 9504 & (00000100001) & 7 \\
\hline 924 & $(10000000002)$ & $\neq 13$ & 4863 & $(01000000002)$ & 3 & 10296 & (00000100001) & $\neq 7$ \\
\hline 1221 & $(00000000020)$ & 3 & 5005 & (01000000002) & $\neq 3$ & 11220 & (00000000021) & 3 \\
\hline 1365 & (00000000004) & all & 5148 & (00000001001) & $\neq 5$ & & & \\
\hline 1650 & $(00000000101)$ & 2 & 5500 & (00010000001) & 3 & & & \\
\hline
\end{tabular}

Appendix A.16. Case $A_{12}, M=3000$

(Recall the remark in Appendix A.5.)

\begin{tabular}{rrr||rrr||rrr} 
deg & $\lambda$ & $p$ & deg & $\lambda$ & $p$ & deg & & $\lambda$ \\
\hline 1 & $(000000000000)$ & all & 455 & $(000000000003)$ & all & 1287 & $(000000010000)$ & all \\
13 & $(000000000001)$ & all & 715 & $(000000001000)$ & all & 1651 & $(000000000020)$ & 3 \\
78 & $(000000000010)$ & all & 728 & $(000000000011)$ & $\neq 3$ & 1716 & $(000000100000)$ & all \\
91 & $(000000000002)$ & all & 988 & $(010000000001)$ & 2 & 1820 & $(000000000004)$ & all \\
167 & $(100000000001)$ & 13 & 988 & $(010000000001)$ & 3 & 2288 & $(000000000101)$ & 2 \\
168 & $(100000000001)$ & $\neq 13$ & 1001 & $(010000000001)$ & $\neq 2,3$ & 2366 & $(000000000020)$ & $\neq 3$ \\
286 & $(000000000100)$ & all & 1157 & $(100000000002)$ & 7 & & & \\
442 & $(000000000011)$ & 3 & 1170 & $(100000000002)$ & $\neq 7$ & & &
\end{tabular}

Appendix A.17. Case $A_{13}, M=3000$

(Recall the remark in Appendix A.5.)

\begin{tabular}{rrr||rrr||rrr} 
deg & $\lambda$ & $p$ & deg & deg & $\lambda$ & $p$ & $p$ \\
\hline 1 & $(0000000000000)$ & all & 364 & $(0000000000100)$ & all & 1442 & $(1000000000002)$ & 3 \\
14 & $(0000000000001)$ & all & 546 & $(0000000000011)$ & 3 & 1442 & $(1000000000002)$ & 5 \\
91 & $(0000000000010)$ & all & 560 & $(0000000000003)$ & all & 1456 & $(1000000000002)$ & $\neq 3,5$ \\
105 & $(0000000000002)$ & all & 910 & $(0000000000011)$ & $\neq 3$ & 2002 & $(0000000010000)$ & all \\
194 & $(1000000000001)$ & 2 & 1001 & $(0000000001000)$ & all & 2184 & $(0000000000020)$ & 3 \\
194 & $(1000000000001)$ & 7 & 1246 & $(0100000000001)$ & 13 & 2380 & $(0000000000004)$ & all \\
195 & $(1000000000001)$ & $\neq 2,7$ & 1260 & $(0100000000001)$ & $\neq 13$ & & &
\end{tabular}


Appendix A.18. Case $A_{14}, M=3000$

(Recall the remark in Appendix A.5.)

\begin{tabular}{rrr||rrr||rrr} 
deg & $\lambda$ & $p$ & deg & deg & \multicolumn{2}{c}{$\lambda$} & $p$ \\
\hline 1 & $(00000000000000)$ & all & 224 & $(10000000000001)$ & $\neq 3,5$ & 1545 & $(01000000000001)$ & 2 \\
15 & $(00000000000001)$ & all & 455 & $(00000000000100)$ & all & 1545 & $(01000000000001)$ & 7 \\
105 & $(00000000000010)$ & all & 665 & $(00000000000011)$ & 3 & 1560 & $(01000000000001)$ & $\neq 2,7$ \\
120 & $(00000000000002)$ & all & 680 & $(00000000000003)$ & all & 1785 & $(10000000000002)$ & all \\
223 & $(10000000000001)$ & 3 & 1120 & $(00000000000011)$ & $\neq 3$ & 2835 & $(00000000000020)$ & 3 \\
223 & $(10000000000001)$ & 5 & 1365 & $(00000000001000)$ & all & & &
\end{tabular}

Appendix A.19. Case $A_{15}, M=3000$

(Recall the remark in Appendix A.5.)

\begin{tabular}{rrr||rrr||rrrr} 
deg & $\lambda$ & $p$ & deg & deg & $\lambda$ & $p$ & $p$ \\
\hline 1 & $(000000000000000)$ & all & 560 & $(000000000000100)$ & all & 1888 & $(010000000000001)$ & 5 \\
16 & $(000000000000001)$ & all & 800 & $(000000000000011)$ & 3 & 1904 & $(010000000000001)$ & $\neq 3,5$ \\
120 & $(000000000000010)$ & all & 816 & $(000000000000003)$ & all & 2144 & $(100000000000002)$ & 17 \\
136 & $(000000000000002)$ & all & 1360 & $(000000000000011)$ & $\neq 3$ & 2160 & $(100000000000002)$ & $\neq 17$ \\
254 & $(100000000000001)$ & 2 & 1820 & $(000000000001000)$ & all & & & \\
255 & $(100000000000001)$ & $\neq 2$ & 1888 & $(010000000000001)$ & 3 & & &
\end{tabular}

Appendix A.20. Case $A_{16}, M=3000$

(Recall the remark in Appendix A.5.)

\begin{tabular}{rrr||rrr||rrr} 
deg & $\lambda$ & $p$ & deg & deg & $\lambda$ & $p$ & $\lambda$ & $p$ \\
\hline 1 & $(0000000000000000)$ & all & 288 & $(1000000000000001)$ & $\neq 17$ & 2278 & $(0100000000000001)$ & 2 \\
17 & $(0000000000000001)$ & all & 680 & $(0000000000000100)$ & all & 2295 & $(0100000000000001)$ & $\neq 2$ \\
136 & $(0000000000000010)$ & all & 952 & $(0000000000000011)$ & 3 & 2380 & $(0000000000001000)$ & all \\
153 & $(0000000000000002)$ & all & 969 & $(0000000000000003)$ & all & 2567 & $(1000000000000002)$ & 3 \\
287 & $(1000000000000001)$ & 17 & 1632 & $(0000000000000011)$ & $\neq 3$ & 2584 & $(1000000000000002)$ & $\neq 3$
\end{tabular}

Appendix A.21. Case $A_{17}, M=3000$

(Recall the remark in Appendix A.5.)

\begin{tabular}{rrr||rrr} 
deg & $\lambda$ & $p$ & deg & $\lambda$ & $p$ \\
\hline 1 & $(00000000000000000)$ & all & 816 & $(00000000000000100)$ & all \\
18 & $(00000000000000001)$ & all & 1122 & $(00000000000000011)$ & 3 \\
153 & $(00000000000000010)$ & all & 1140 & $(00000000000000003)$ & all \\
171 & $(00000000000000002)$ & all & 1938 & $(00000000000000011)$ & $\neq 3$ \\
322 & $(10000000000000001)$ & 2 & 2718 & $(01000000000000001)$ & 17 \\
322 & $(10000000000000001)$ & 3 & 2736 & $(01000000000000001)$ & $\neq 17$ \\
323 & $(10000000000000001)$ & $\neq 2,3$ & & &
\end{tabular}


Appendix A.22. Case $B_{2}, M=300$

\begin{tabular}{|c|c|c|c|c|c|c|c|c|}
\hline deg & $\lambda$ & $p$ & deg & $\lambda$ & $p$ & deg & $\lambda$ & $p$ \\
\hline 1 & $(00)$ & all & 81 & (22) & $\neq 5,7$ & 199 & (07) & 11 \\
\hline 4 & (01) & 2 & 84 & (15) & 13 & 199 & (25) & 7 \\
\hline 4 & (10) & all & 84 & (60) & all & 200 & (71) & 11 \\
\hline 5 & (01) & $\neq 2$ & 85 & (06) & 13 & 204 & (07) & $\neq 11,13$ \\
\hline 10 & (20) & all & 86 & (23) & 5 & 204 & (33) & 5 \\
\hline 12 & (11) & 5 & 91 & (05) & $\neq 11$ & 206 & (24) & 7 \\
\hline 13 & (02) & 5 & 105 & (41) & all & 220 & (42) & $\neq 5,7$ \\
\hline 14 & (02) & $\neq 5$ & 115 & (42) & 5 & 220 & (90) & all \\
\hline 16 & (11) & $\neq 5$ & 116 & (33) & 11 & 224 & (15) & $\neq 11,13$ \\
\hline 20 & (30) & all & 116 & (51) & 7 & 231 & (61) & all \\
\hline 24 & (12) & 7 & 120 & (70) & all & 236 & (53) & 13 \\
\hline 25 & (03) & 7 & 126 & (06) & 11 & 251 & (25) & 13 \\
\hline 25 & (21) & 3 & 140 & (06) & $\neq 11,13$ & 256 & (16) & 13 \\
\hline 30 & (03) & $\neq 7$ & 140 & (14) & $\neq 11$ & 256 & (33) & $\neq 5,7,11$ \\
\hline 35 & (21) & $\neq 3$ & 140 & (32) & all & 260 & (24) & $\neq 7,11$ \\
\hline 35 & (40) & all & 144 & (17) & 17 & 260 & (44) & 7 \\
\hline 40 & (12) & $\neq 7$ & 145 & (08) & 17 & 264 & $(1,10)$ & 23 \\
\hline 44 & (31) & 7 & 149 & (42) & 7 & 265 & $(0,11)$ & 23 \\
\hline 52 & (31) & 5 & 154 & (23) & $\neq 5$ & 271 & (08) & 13 \\
\hline 54 & (04) & 7 & 160 & (51) & $\neq 7$ & 284 & (08) & 11 \\
\hline 55 & (04) & $\neq 7$ & 164 & (34) & 13 & 284 & (36) & 17 \\
\hline 56 & (50) & all & 164 & (52) & 11 & 285 & (08) & $\neq 11,13,17$ \\
\hline 60 & (14) & 11 & 165 & (80) & all & 285 & (43) & 11 \\
\hline 61 & (05) & 11 & 174 & (07) & 13 & 286 & $(10,0)$ & all \\
\hline 64 & (31) & $\neq 5,7$ & 179 & (24) & 11 & 294 & (09) & 17 \\
\hline 68 & (22) & 5 & 180 & (18) & 19 & 296 & (72) & 13 \\
\hline 71 & (22) & 7 & 180 & (33) & 7 & 300 & (52) & 7 \\
\hline 76 & (13) & 7 & 181 & (09) & 19 & & & \\
\hline 80 & (13) & $\neq 7$ & 184 & (15) & 11 & & & \\
\hline
\end{tabular}

Appendix A.23. Case $B_{3}, M=700$

\begin{tabular}{rrr||rrr||rrr} 
deg & $\lambda$ & $p$ & deg & $\lambda$ & $p$ & deg & $\lambda$ & $p$ \\
\hline 1 & $(000)$ & all & 141 & $(020)$ & 5 & 384 & $(111)$ & 3 \\
6 & $(001)$ & 2 & 155 & $(004)$ & 11 & 448 & $(103)$ & $\neq 11$ \\
7 & $(001)$ & $\neq 2$ & 168 & $(020)$ & $\neq 3,5$ & 448 & $(202)$ & 5 \\
8 & $(100)$ & all & 168 & $(102)$ & $\neq 3$ & 448 & $(310)$ & 7 \\
14 & $(010)$ & 2 & 168 & $(201)$ & 5 & 472 & $(111)$ & 7 \\
21 & $(010)$ & $\neq 2$ & 182 & $(004)$ & $\neq 11$ & 483 & $(022)$ & 5 \\
26 & $(002)$ & 7 & 189 & $(201)$ & $\neq 5$ & 512 & $(111)$ & $\neq 3,5,7$ \\
27 & $(002)$ & $\neq 7$ & 189 & $(210)$ & 3 & 518 & $(202)$ & 3 \\
35 & $(200)$ & all & 248 & $(120)$ & 7 & 521 & $(030)$ & 7 \\
40 & $(101)$ & 7 & 280 & $(103)$ & 11 & 560 & $(104)$ & 13 \\
48 & $(101)$ & $\neq 7$ & 293 & $(400)$ & 5 & 560 & $(301)$ & all \\
63 & $(011)$ & 3 & 294 & $(400)$ & $\neq 5$ & 560 & $(500)$ & 7 \\
64 & $(011)$ & 2 & 301 & $(005)$ & 13 & 616 & $(021)$ & 5 \\
64 & $(110)$ & 5 & 304 & $(012)$ & 7 & 616 & $(202)$ & $\neq 3,5$ \\
77 & $(003)$ & all & 309 & $(012)$ & 3 & 664 & $(310)$ & 5 \\
104 & $(110)$ & 3 & 330 & $(012)$ & $\neq 3,7$ & 672 & $(021)$ & 3 \\
104 & $(300)$ & 5 & 344 & $(111)$ & 5 & 672 & $(120)$ & 3 \\
105 & $(011)$ & $\neq 2,3$ & 371 & $(005)$ & 11 & 672 & $(500)$ & $\neq 7$ \\
112 & $(110)$ & $\neq 3,5$ & 371 & $(013)$ & 5 & 687 & $(006)$ & 13 \\
112 & $(300)$ & $\neq 5$ & 371 & $(210)$ & 5 & 693 & $(021)$ & $\neq 3,5$ \\
120 & $(102)$ & 3 & 378 & $(005)$ & $\neq 11,13$ & & & \\
132 & $(020)$ & 3 & 378 & $(210)$ & $\neq 3,5$ & & &
\end{tabular}


Appendix A.24. Case $B_{4}, M=1000$

\begin{tabular}{rrr||rrr||rrr} 
deg & $\lambda$ & $p$ & deg & $\lambda$ & $p$ & deg & $\lambda$ & $p$ \\
\hline 1 & $(0000)$ & all & 160 & $(0011)$ & 2 & 576 & $(1002)$ & $\neq 11$ \\
8 & $(0001)$ & 2 & 231 & $(0011)$ & $\neq 2,3$ & 579 & $(0110)$ & 3 \\
9 & $(0001)$ & $\neq 2$ & 246 & $(0101)$ & 2 & 594 & $(0101)$ & $\neq 2,7$ \\
16 & $(1000)$ & all & 304 & $(1010)$ & 7 & 656 & $(1100)$ & 3 \\
26 & $(0010)$ & 2 & 336 & $(1100)$ & 5 & 672 & $(3000)$ & $\neq 5$ \\
36 & $(0010)$ & $\neq 2$ & 369 & $(0020)$ & 3 & 752 & $(1100)$ & 7 \\
43 & $(0002)$ & 3 & 406 & $(0004)$ & 13 & 768 & $(1100)$ & $\neq 3,5,7$ \\
44 & $(0002)$ & $\neq 3$ & 416 & $(1010)$ & 2 & 784 & $(0110)$ & 2 \\
48 & $(0100)$ & 2 & 432 & $(1010)$ & $\neq 2,7$ & 798 & $(2001)$ & 5 \\
84 & $(0100)$ & $\neq 2$ & 448 & $(1002)$ & 11 & 840 & $(2001)$ & 3 \\
112 & $(1001)$ & 3 & 449 & $(0004)$ & 11 & 867 & $(0012)$ & 3 \\
126 & $(2000)$ & all & 450 & $(0004)$ & $\neq 11,13$ & 874 & $(0012)$ & 11 \\
128 & $(1001)$ & $\neq 3$ & 451 & $(0020)$ & 7 & 910 & $(0012)$ & $\neq 3,11$ \\
147 & $(0003)$ & 11 & 495 & $(0020)$ & $\neq 3,7$ & 924 & $(2001)$ & $\neq 3,5$ \\
147 & $(0011)$ & 3 & 544 & $(3000)$ & 5 & 957 & $(0013)$ & 5 \\
156 & $(0003)$ & $\neq 11$ & 558 & $(0101)$ & 7 & & &
\end{tabular}

Appendix A.25. Case $B_{5}, M=2000$

\begin{tabular}{rrr||rrr||rrr} 
deg & $\lambda$ & $p$ & $\operatorname{deg}$ & $\lambda$ & $p$ & $\operatorname{deg}$ & $\lambda$ & $p$ \\
\hline 1 & $(00000)$ & all & 288 & $(10001)$ & 11 & 1144 & $(00020)$ & $\neq 3,5$ \\
10 & $(00001)$ & 2 & 320 & $(00011)$ & 2 & 1375 & $(00101)$ & 3 \\
11 & $(00001)$ & $\neq 2$ & 320 & $(10001)$ & $\neq 11$ & 1376 & $(10010)$ & 5 \\
32 & $(10000)$ & all & 330 & $(01000)$ & $\neq 2$ & 1408 & $(01001)$ & 2 \\
44 & $(00010)$ & 2 & 418 & $(00011)$ & 5 & 1408 & $(10010)$ & $\neq 3,5$ \\
55 & $(00010)$ & $\neq 2$ & 429 & $(00011)$ & $\neq 2,3,5$ & 1430 & $(00101)$ & $\neq 2,3$ \\
64 & $(00002)$ & 11 & 462 & $(20000)$ & all & 1440 & $(10002)$ & 13 \\
65 & $(00002)$ & $\neq 11$ & 670 & $(00101)$ & 2 & 1760 & $(10002)$ & $\neq 13$ \\
100 & $(00100)$ & 2 & 749 & $(00020)$ & 3 & 1760 & $(11000)$ & 5 \\
164 & $(01000)$ & 2 & 870 & $(00004)$ & 5 & 1961 & $(00012)$ & 11 \\
165 & $(00100)$ & $\neq 2$ & 934 & $(00004)$ & 13 & 1970 & $(00012)$ & 13 \\
264 & $(00003)$ & 13 & 935 & $(00004)$ & $\neq 5,13$ & 1991 & $(00110)$ & 3 \\
264 & $(00011)$ & 3 & 1088 & $(10010)$ & 3 & & & \\
275 & $(00003)$ & $\neq 13$ & 1143 & $(00020)$ & 5 & & &
\end{tabular}

Appendix A.26. Case $B_{6}, M=4000$

\begin{tabular}{rrr||rrr||rrr} 
deg & $\lambda$ & $p$ & $\operatorname{deg}$ & $\lambda$ & $p$ & $\operatorname{deg}$ & $\lambda$ & $p$ \\
\hline 1 & $(000000)$ & all & 442 & $(000003)$ & $\neq 5$ & 1729 & $(000004)$ & $\neq 5,17$ \\
12 & $(000001)$ & 2 & 560 & $(000011)$ & 2 & 2185 & $(000020)$ & 11 \\
13 & $(000001)$ & $\neq 2$ & 560 & $(010000)$ & 2 & 2275 & $(000020)$ & $\neq 3,11$ \\
64 & $(000010)$ & 2 & 704 & $(100001)$ & 13 & 2847 & $(000101)$ & 11 \\
64 & $(100000)$ & all & 715 & $(000011)$ & $\neq 2,3$ & 2925 & $(000101)$ & $\neq 2,11$ \\
78 & $(000010)$ & $\neq 2$ & 715 & $(001000)$ & $\neq 2$ & 3392 & $(100010)$ & 11 \\
89 & $(000002)$ & 13 & 768 & $(100001)$ & $\neq 13$ & 3808 & $(001001)$ & 2 \\
90 & $(000002)$ & $\neq 13$ & 1287 & $(010000)$ & $\neq 2$ & 3838 & $(000012)$ & 13 \\
208 & $(000100)$ & 2 & 1508 & $(000101)$ & 2 & 3849 & $(000012)$ & 3 \\
286 & $(000100)$ & $\neq 2$ & 1559 & $(000020)$ & 3 & 3849 & $(000012)$ & 5 \\
364 & $(001000)$ & 2 & 1639 & $(000004)$ & 17 & 3927 & $(000012)$ & $\neq 3,5,13$ \\
416 & $(000011)$ & 3 & 1716 & $(200000)$ & all & & & \\
429 & $(000003)$ & 5 & 1728 & $(000004)$ & 5 & & &
\end{tabular}


Appendix A.27. Case $B_{7}, M=5000$

\begin{tabular}{rrr||rrr||rrr} 
deg & $\lambda$ & $p$ & deg & $\lambda$ & $p$ & deg & $\lambda$ & $p$ \\
\hline 1 & $(0000000)$ & all & 650 & $(0000003)$ & 17 & 1792 & $(1000001)$ & $\neq 3,5$ \\
14 & $(0000001)$ & 2 & 650 & $(0000011)$ & 3 & 1912 & $(0100000)$ & 2 \\
15 & $(0000001)$ & $\neq 2$ & 665 & $(0000003)$ & $\neq 17$ & 2715 & $(0000020)$ & 3 \\
90 & $(0000010)$ & 2 & 896 & $(0000011)$ & 2 & 2821 & $(0000004)$ & 19 \\
105 & $(0000010)$ & $\neq 2$ & 910 & $(0001000)$ & 2 & 2884 & $(0000101)$ & 2 \\
118 & $(0000002)$ & 3 & 1090 & $(0000011)$ & 7 & 2939 & $(0000004)$ & 17 \\
118 & $(0000002)$ & 5 & 1105 & $(0000011)$ & $\neq 2,3,7$ & 2940 & $(0000004)$ & $\neq 17,19$ \\
119 & $(0000002)$ & $\neq 3,5$ & 1288 & $(0010000)$ & 2 & 3003 & $(0010000)$ & $\neq 2$ \\
128 & $(1000000)$ & all & 1365 & $(0001000)$ & $\neq 2$ & 3961 & $(0000020)$ & 13 \\
336 & $(0000100)$ & 2 & 1664 & $(1000001)$ & 3 & 4079 & $(0000020)$ & 7 \\
455 & $(0000100)$ & $\neq 2$ & 1664 & $(1000001)$ & 5 & 4080 & $(0000020)$ & $\neq 3,7,13$
\end{tabular}

Appendix A.28. Case $B_{8}, M=7000$

\begin{tabular}{rrr||rrr||rrr} 
deg & $\lambda$ & $p$ & deg & $\lambda$ & $p$ & deg & $\lambda$ & $p$ \\
\hline 1 & $(00000000)$ & all & 935 & $(00000003)$ & 19 & 4251 & $(00000020)$ & 3 \\
16 & $(00000001)$ & 2 & 935 & $(00000011)$ & 3 & 4488 & $(00100000)$ & 2 \\
17 & $(00000001)$ & $\neq 2$ & 952 & $(00000003)$ & $\neq 19$ & 4540 & $(00000004)$ & 7 \\
118 & $(00000010)$ & 2 & 1344 & $(00000011)$ & 2 & 4691 & $(00000004)$ & 19 \\
136 & $(00000010)$ & $\neq 2$ & 1582 & $(00001000)$ & 2 & 4692 & $(00000004)$ & $\neq 7,19$ \\
151 & $(00000002)$ & 17 & 1615 & $(00000011)$ & $\neq 2,3$ & 5066 & $(00000101)$ & 2 \\
152 & $(00000002)$ & $\neq 17$ & 2380 & $(00001000)$ & $\neq 2$ & 6188 & $(00010000)$ & $\neq 2$ \\
256 & $(10000000)$ & all & 3808 & $(00010000)$ & 2 & 6528 & $(01000000)$ & 2 \\
544 & $(00000100)$ & 2 & 3840 & $(10000001)$ & 17 & 6631 & $(00000020)$ & 5 \\
680 & $(00000100)$ & $\neq 2$ & 4096 & $(10000001)$ & $\neq 17$ & 6783 & $(00000020)$ & $\neq 3,5$
\end{tabular}

Appendix A.29. Case $B_{9}, M=8000$

\begin{tabular}{rrr||rrr||rrr} 
deg & $\lambda$ & $p$ & deg & $\lambda$ & $p$ & deg & $\lambda$ & $p$ \\
\hline 1 & $(000000000)$ & all & 780 & $(000000100)$ & 2 & 3876 & $(000001000)$ & $\neq 2$ \\
18 & $(000000001)$ & 2 & 969 & $(000000100)$ & $\neq 2$ & 6763 & $(000000020)$ & 3 \\
19 & $(000000001)$ & $\neq 2$ & 1273 & $(000000011)$ & 3 & 6936 & $(000000004)$ & 23 \\
152 & $(000000010)$ & 2 & 1292 & $(000000003)$ & 7 & 6972 & $(000010000)$ & 2 \\
171 & $(000000010)$ & $\neq 2$ & 1311 & $(000000003)$ & $\neq 7$ & 7124 & $(000000004)$ & 7 \\
188 & $(000000002)$ & 19 & 1920 & $(000000011)$ & 2 & 7125 & $(000000004)$ & $\neq 7,23$ \\
189 & $(000000002)$ & $\neq 19$ & 2261 & $(000000011)$ & $\neq 2,3$ & & & \\
512 & $(100000000)$ & all & 2906 & $(000001000)$ & 2 & & &
\end{tabular}

Appendix A.30. Case $B_{10}, M=10000$

\begin{tabular}{rrr||rrr||rrr} 
deg & $\lambda$ & $p$ & deg & $\lambda$ & $p$ & deg & $\lambda$ & $p$ \\
\hline 1 & $(0000000000)$ & all & 230 & $(0000000002)$ & $\neq 3,7$ & 2640 & $(0000000011)$ & 2 \\
20 & $(0000000001)$ & 2 & 1024 & $(1000000000)$ & all & 3038 & $(0000000011)$ & 5 \\
21 & $(0000000001)$ & $\neq 2$ & 1120 & $(0000000100)$ & 2 & 3059 & $(0000000011)$ & $\neq 2,3,5$ \\
188 & $(0000000010)$ & 2 & 1330 & $(0000000100)$ & $\neq 2$ & 4466 & $(0000001000)$ & 2 \\
210 & $(0000000010)$ & $\neq 2$ & 1729 & $(0000000003)$ & 23 & 5985 & $(0000001000)$ & $\neq 2$ \\
229 & $(0000000002)$ & 3 & 1729 & $(0000000011)$ & 3 & 9954 & $(0000000020)$ & 3 \\
229 & $(0000000002)$ & 7 & 1750 & $(0000000003)$ & $\neq 23$ & & &
\end{tabular}

Appendix A.31. Case $B_{11}, M=12000$

\begin{tabular}{rrr||rrr||rrr} 
deg & $\lambda$ & $p$ & $\operatorname{deg}$ & \multicolumn{1}{c}{$\lambda$} & $p$ & $\operatorname{deg}$ & $\lambda$ & $p$ \\
\hline 1 & $(00000000000)$ & all & 275 & $(00000000002)$ & $\neq 23$ & 2277 & $(00000000003)$ & $\neq 5$ \\
22 & $(00000000001)$ & 2 & 1496 & $(00000000100)$ & 2 & 3520 & $(00000000011)$ & 2 \\
23 & $(00000000001)$ & $\neq 2$ & 1771 & $(00000000100)$ & $\neq 2$ & 4002 & $(00000000011)$ & 11 \\
230 & $(00000000010)$ & 2 & 2048 & $(10000000000)$ & all & 4025 & $(00000000011)$ & $\neq 2,3,11$ \\
253 & $(00000000010)$ & $\neq 2$ & 2254 & $(00000000003)$ & 5 & 7084 & $(00000001000)$ & 2 \\
274 & $(00000000002)$ & 23 & 2254 & $(00000000011)$ & 3 & 8855 & $(00000001000)$ & $\neq 2$
\end{tabular}


Appendix A.32. Case $C_{3}, M=1000$

\begin{tabular}{rrr||rrr||rrr} 
deg & $\lambda$ & $p$ & deg & $\lambda$ & $p$ & deg & $\lambda$ & $p$ \\
\hline 1 & $(000)$ & all & 172 & $(300)$ & 7 & 594 & $(210)$ & $\neq 5,7$ \\
6 & $(001)$ & all & 189 & $(012)$ & all & 616 & $(120)$ & $\neq 3,5$ \\
8 & $(100)$ & 2 & 202 & $(102)$ & 5 & 623 & $(202)$ & 3 \\
13 & $(010)$ & 3 & 216 & $(102)$ & $\neq 5,7$ & 665 & $(410)$ & 11 \\
14 & $(010)$ & $\neq 3$ & 246 & $(013)$ & 5 & 666 & $(031)$ & 5 \\
14 & $(100)$ & $\neq 2$ & 252 & $(005)$ & all & 666 & $(500)$ & 11 \\
21 & $(002)$ & all & 286 & $(021)$ & 3 & 786 & $(015)$ & 7 \\
48 & $(101)$ & 2 & 295 & $(030)$ & 5 & 792 & $(007)$ & all \\
50 & $(011)$ & 3 & 309 & $(111)$ & 5 & 798 & $(014)$ & 5 \\
56 & $(003)$ & all & 316 & $(120)$ & 3 & 813 & $(301)$ & 5 \\
57 & $(101)$ & 3 & 316 & $(201)$ & 5 & 840 & $(130)$ & 7 \\
58 & $(011)$ & 7 & 330 & $(300)$ & $\neq 7$ & 854 & $(211)$ & 7 \\
62 & $(110)$ & 5 & 350 & $(021)$ & $\neq 3$ & 861 & $(301)$ & 7 \\
63 & $(200)$ & 5 & 358 & $(111)$ & 3 & 875 & $(400)$ & 7 \\
64 & $(011)$ & $\neq 3,7$ & 378 & $(201)$ & $\neq 5$ & 903 & $(022)$ & 3 \\
70 & $(101)$ & $\neq 2,3$ & 385 & $(030)$ & $\neq 5$ & 924 & $(014)$ & $\neq 5$ \\
84 & $(200)$ & $\neq 5$ & 423 & $(111)$ & 7 & 924 & $(022)$ & $\neq 3,5$ \\
89 & $(020)$ & 7 & 426 & $(022)$ & 5 & 924 & $(112)$ & 5 \\
90 & $(020)$ & $\neq 7$ & 448 & $(013)$ & $\neq 5$ & 938 & $(400)$ & 5 \\
112 & $(110)$ & 2 & 462 & $(006)$ & all & 942 & $(104)$ & 7 \\
126 & $(004)$ & all & 512 & $(111)$ & $\neq 3,5,7$ & 951 & $(040)$ & 5 \\
126 & $(110)$ & $\neq 2,5$ & 525 & $(103)$ & all & 951 & $(202)$ & 5 \\
158 & $(102)$ & 7 & 552 & $(120)$ & 5 & 994 & $(031)$ & 11 \\
171 & $(210)$ & 7 & 573 & $(210)$ & 5 & & & \\
& & & & & & & &
\end{tabular}

Appendix A.33. Case $C_{4}, M=2000$

\begin{tabular}{rrr||rrr||rrr} 
deg & $\lambda$ & $p$ & deg & $\lambda$ & $p$ & deg & $\lambda$ & $p$ \\
\hline 1 & $(0000)$ & all & 312 & $(1100)$ & 5 & 792 & $(1010)$ & $\neq 2,3,7$ \\
8 & $(0001)$ & all & 313 & $(2000)$ & 5 & 825 & $(0200)$ & $\neq 3,7$ \\
16 & $(1000)$ & 2 & 315 & $(0101)$ & $\neq 2,3$ & 944 & $(0102)$ & 5 \\
26 & $(0010)$ & 2 & 330 & $(0004)$ & all & 1016 & $(1100)$ & 3 \\
27 & $(0010)$ & $\neq 2$ & 416 & $(1010)$ & 2 & 1048 & $(1100)$ & 7 \\
36 & $(0002)$ & all & 504 & $(0110)$ & 3 & 1056 & $(1100)$ & $\neq 2,3,5,7$ \\
40 & $(0100)$ & 3 & 513 & $(1010)$ & 3 & 1072 & $(0102)$ & 3 \\
41 & $(1000)$ & 3 & 558 & $(0012)$ & 5 & 1114 & $(1002)$ & 3 \\
42 & $(1000)$ & $\neq 2,3$ & 593 & $(2000)$ & 7 & 1155 & $(1002)$ & $\neq 3$ \\
48 & $(0100)$ & $\neq 3$ & 594 & $(0012)$ & $\neq 5$ & 1200 & $(2100)$ & 7 \\
112 & $(0011)$ & 3 & 594 & $(2000)$ & $\neq 5,7$ & 1201 & $(3000)$ & 7 \\
120 & $(0003)$ & all & 632 & $(0110)$ & 7 & 1232 & $(0102)$ & $\neq 3,5$ \\
128 & $(1001)$ & 2 & 744 & $(0110)$ & 5 & 1352 & $(0021)$ & 11 \\
160 & $(0011)$ & $\neq 3$ & 765 & $(1010)$ & 7 & 1504 & $(0021)$ & 5 \\
240 & $(1001)$ & 7 & 768 & $(1100)$ & 2 & 1512 & $(0021)$ & $\neq 5,11$ \\
246 & $(0101)$ & 2 & 784 & $(0013)$ & 5 & 1608 & $(0013)$ & 11 \\
266 & $(0020)$ & 3 & 784 & $(0110)$ & 2 & 1652 & $(0022)$ & 5 \\
279 & $(0101)$ & 3 & 784 & $(0200)$ & 3 & 1716 & $(0006)$ & all \\
281 & $(0020)$ & 5 & 789 & $(0200)$ & 7 & 1728 & $(0013)$ & $\neq 5,11$ \\
288 & $(1001)$ & $\neq 2,7$ & 792 & $(0005)$ & all & 1891 & $(0111)$ & 3 \\
308 & $(0020)$ & $\neq 3,5$ & 792 & $(0110)$ & $\neq 2,3,5,7$ & & &
\end{tabular}


Small degree representations in defining characteristic

Appendix A.34. Case $C_{5}, M=2500$

\begin{tabular}{rrr||rrr||rrr} 
deg & $\lambda$ & $p$ & deg & $\lambda$ & $p$ & deg & $\lambda$ & $p$ \\
\hline 1 & $(00000)$ & all & 210 & $(00011)$ & 3 & 1099 & $(10001)$ & 3 \\
10 & $(00001)$ & all & 220 & $(00003)$ & all & 1155 & $(10001)$ & $\neq 2,3$ \\
32 & $(10000)$ & 2 & 310 & $(00011)$ & 11 & 1276 & $(01001)$ & 5 \\
43 & $(00010)$ & 5 & 320 & $(00011)$ & $\neq 3,11$ & 1375 & $(00012)$ & 3 \\
44 & $(00010)$ & $\neq 5$ & 320 & $(10001)$ & 2 & 1408 & $(01001)$ & $\neq 3,5$ \\
55 & $(00002)$ & all & 615 & $(00020)$ & 3 & 1408 & $(10010)$ & 2 \\
100 & $(00100)$ & 2 & 670 & $(00101)$ & 2 & 1430 & $(00012)$ & $\neq 3$ \\
110 & $(00100)$ & $\neq 2$ & 715 & $(00004)$ & all & 1452 & $(00110)$ & 3 \\
121 & $(01000)$ & 3 & 779 & $(00020)$ & 11 & 1562 & $(11000)$ & 5 \\
122 & $(10000)$ & 3 & 780 & $(00020)$ & $\neq 3,11$ & 1563 & $(20000)$ & 5 \\
132 & $(10000)$ & $\neq 2,3$ & 848 & $(00101)$ & 5 & 1992 & $(00013)$ & 5 \\
164 & $(01000)$ & 2 & 891 & $(00101)$ & $\neq 2,5$ & 2002 & $(00005)$ & all \\
165 & $(01000)$ & $\neq 2,3$ & 1088 & $(01001)$ & 3 & & &
\end{tabular}

Appendix A.35. Case $C_{6}, M=4000$

\begin{tabular}{rrr||rrr||rrr} 
deg & $\lambda$ & $p$ & deg & $\lambda$ & $p$ & deg & $\lambda$ & $p$ \\
\hline 1 & $(000000)$ & all & 365 & $(100000)$ & 3 & 1649 & $(000020)$ & 13 \\
12 & $(000001)$ & all & 428 & $(001000)$ & 5 & 1650 & $(000020)$ & $\neq 3,7,13$ \\
64 & $(000010)$ & 2 & 429 & $(001000)$ & $\neq 2,5$ & 1924 & $(000101)$ & 5 \\
64 & $(000010)$ & 3 & 429 & $(100000)$ & $\neq 2,3$ & 1938 & $(000101)$ & 3 \\
64 & $(100000)$ & 2 & 548 & $(000011)$ & 13 & 2002 & $(000101)$ & $\neq 2,3,5$ \\
65 & $(000010)$ & $\neq 2,3$ & 560 & $(000011)$ & $\neq 3,13$ & 2847 & $(000012)$ & 7 \\
78 & $(000002)$ & all & 560 & $(010000)$ & 2 & 2925 & $(000012)$ & $\neq 7$ \\
196 & $(000100)$ & 5 & 572 & $(010000)$ & $\neq 2,3$ & 3432 & $(000110)$ & 3 \\
208 & $(000100)$ & $\neq 5$ & 768 & $(100001)$ & 2 & 3638 & $(010001)$ & 3 \\
352 & $(000011)$ & 3 & 1221 & $(000020)$ & 3 & 3652 & $(100001)$ & 3 \\
364 & $(000003)$ & all & 1365 & $(000004)$ & all & 3796 & $(001001)$ & 5 \\
364 & $(001000)$ & 2 & 1508 & $(000101)$ & 2 & 3808 & $(001001)$ & 2 \\
364 & $(010000)$ & 3 & 1585 & $(000020)$ & 7 & & &
\end{tabular}

Appendix A.36. Case $C_{7}, M=6000$

\begin{tabular}{rrr||rrr||rrr} 
deg & $\lambda$ & $p$ & deg & $\lambda$ & $p$ & deg & $\lambda$ & $p$ \\
\hline 1 & $(0000000)$ & all & 882 & $(0000011)$ & 5 & 2001 & $(0100000)$ & 5 \\
14 & $(0000001)$ & all & 896 & $(0000011)$ & $\neq 3,5$ & 2002 & $(0100000)$ & $\neq 2,3,5$ \\
89 & $(0000010)$ & 7 & 909 & $(0001000)$ & 3 & 2184 & $(0000020)$ & 3 \\
90 & $(0000010)$ & $\neq 7$ & 910 & $(0001000)$ & $\neq 3,5$ & 2380 & $(0000004)$ & all \\
105 & $(0000002)$ & all & 1093 & $(0100000)$ & 3 & 2884 & $(0000101)$ & 2 \\
128 & $(1000000)$ & 2 & 1094 & $(1000000)$ & 3 & 3093 & $(0000020)$ & 5 \\
336 & $(0000100)$ & 2 & 1288 & $(0010000)$ & 2 & 3094 & $(0000020)$ & $\neq 3,5$ \\
336 & $(0000100)$ & 3 & 1430 & $(1000000)$ & $\neq 2,3$ & 3795 & $(0000101)$ & 3 \\
350 & $(0000100)$ & $\neq 2,3$ & 1624 & $(0010000)$ & 5 & 3811 & $(0000101)$ & 7 \\
546 & $(0000011)$ & 3 & 1638 & $(0010000)$ & $\neq 2,5$ & 3900 & $(0000101)$ & $\neq 2,3,7$ \\
560 & $(0000003)$ & all & 1792 & $(1000001)$ & 2 & 5355 & $(0000012)$ & all \\
820 & $(0001000)$ & 5 & 1912 & $(0100000)$ & 2 & & &
\end{tabular}


Appendix A.37. Case $C_{8}, M=10000$

\begin{tabular}{rrr||rrr||rrr} 
deg & $\lambda$ & $p$ & deg & $\lambda$ & $p$ & deg & $\lambda$ & $p$ \\
\hline 1 & $(00000000)$ & all & 1582 & $(00001000)$ & 2 & 4862 & $(10000000)$ & $\neq 2,3,5$ \\
16 & $(00000001)$ & all & 1699 & $(00001000)$ & 7 & 5066 & $(00000101)$ & 2 \\
118 & $(00000010)$ & 2 & 1700 & $(00001000)$ & $\neq 2,3,7$ & 5319 & $(00000020)$ & 17 \\
119 & $(00000010)$ & $\neq 2$ & 3264 & $(00010000)$ & 5 & 5320 & $(00000020)$ & $\neq 3,17$ \\
136 & $(00000002)$ & all & 3280 & $(01000000)$ & 3 & 6069 & $(00100000)$ & 5 \\
256 & $(10000000)$ & 2 & 3281 & $(10000000)$ & 3 & 6188 & $(00100000)$ & $\neq 2,5$ \\
528 & $(00000100)$ & 7 & 3620 & $(00000020)$ & 3 & 6528 & $(01000000)$ & 2 \\
544 & $(00000100)$ & $\neq 7$ & 3792 & $(00010000)$ & 3 & 6749 & $(00000101)$ & 7 \\
800 & $(00000011)$ & 3 & 3808 & $(00010000)$ & $\neq 3,5$ & 6885 & $(00000101)$ & $\neq 2,7$ \\
816 & $(00000003)$ & all & 3876 & $(00000004)$ & all & 7056 & $(01000000)$ & 5 \\
1328 & $(00000011)$ & 17 & 4096 & $(10000001)$ & 2 & 7072 & $(01000000)$ & $\neq 2,3,5$ \\
1344 & $(00000011)$ & $\neq 3,17$ & 4488 & $(00100000)$ & 2 & 8908 & $(00000012)$ & 3 \\
1581 & $(00001000)$ & 3 & 4861 & $(10000000)$ & 5 & 9044 & $(00000012)$ & $\neq 3$
\end{tabular}

Appendix A.38. Case $C_{9}, M=10000$

\begin{tabular}{rrr||rrr||rrr} 
deg & $\lambda$ & $p$ & deg & $\lambda$ & $p$ & deg & $\lambda$ & $p$ \\
\hline 1 & $(000000000)$ & all & 1902 & $(000000011)$ & 19 & 7752 & $(000010000)$ & $\neq 2,3,7$ \\
18 & $(000000001)$ & all & 1920 & $(000000011)$ & $\neq 3,19$ & 8226 & $(000000101)$ & 2 \\
151 & $(000000010)$ & 3 & 2755 & $(000001000)$ & 7 & 8416 & $(000000020)$ & 5 \\
152 & $(000000010)$ & $\neq 3$ & 2906 & $(000001000)$ & 2 & 8567 & $(000000020)$ & 19 \\
171 & $(000000002)$ & all & 2907 & $(000001000)$ & $\neq 2,7$ & 8568 & $(000000020)$ & $\neq 3,5,19$ \\
512 & $(100000000)$ & 2 & 5661 & $(000000020)$ & 3 & 9216 & $(100000001)$ & 2 \\
780 & $(000000100)$ & 2 & 5985 & $(000000004)$ & all & 9841 & $(010000000)$ & 3 \\
798 & $(000000100)$ & $\neq 2$ & 6954 & $(000010000)$ & 3 & 9842 & $(100000000)$ & 3 \\
1122 & $(000000011)$ & 3 & 6972 & $(000010000)$ & 2 & & & \\
1140 & $(000000003)$ & all & 7734 & $(000010000)$ & 7 & & &
\end{tabular}

Appendix A.39. Case $C_{10}, M=10000$

\begin{tabular}{rrr||rrr||rrr} 
deg & $\lambda$ & $p$ & $\operatorname{deg}$ & $\lambda$ & $p$ & $\operatorname{deg}$ & $\lambda$ & $p$ \\
\hline 1 & $(0000000000)$ & all & 1024 & $(1000000000)$ & 2 & 2640 & $(0000000011)$ & $\neq 3,7$ \\
20 & $(0000000001)$ & all & 1100 & $(0000000100)$ & 3 & 4466 & $(0000001000)$ & 2 \\
188 & $(0000000010)$ & 2 & 1120 & $(0000000100)$ & $\neq 3$ & 4654 & $(0000001000)$ & 3 \\
188 & $(0000000010)$ & 5 & 1520 & $(0000000011)$ & 3 & 4655 & $(0000001000)$ & $\neq 2,3$ \\
189 & $(0000000010)$ & $\neq 2,5$ & 1540 & $(0000000003)$ & all & 8455 & $(0000000020)$ & 3 \\
210 & $(0000000002)$ & all & 2620 & $(0000000011)$ & 7 & 8855 & $(0000000004)$ & all
\end{tabular}

Appendix A.40. Case $C_{11}, M=12000$

\begin{tabular}{rrr||rrr||rrr} 
deg & $\lambda$ & $p$ & deg & $\lambda$ & $p$ & deg & $\lambda$ & $p$ \\
\hline 1 & $(00000000000)$ & all & 1496 & $(00000000100)$ & 5 & 3520 & $(00000000011)$ & $\neq 3,23$ \\
22 & $(00000000001)$ & all & 1518 & $(00000000100)$ & $\neq 2,5$ & 6854 & $(00000001000)$ & 3 \\
229 & $(00000000010)$ & 11 & 2002 & $(00000000011)$ & 3 & 7083 & $(00000001000)$ & 5 \\
230 & $(00000000010)$ & $\neq 11$ & 2024 & $(00000000003)$ & all & 7084 & $(00000001000)$ & $\neq 3,5$ \\
253 & $(00000000002)$ & all & 2048 & $(10000000000)$ & 2 & & & \\
1496 & $(00000000100)$ & 2 & 3498 & $(00000000011)$ & 23 & & &
\end{tabular}


Appendix A.41. Case $D_{4}, M=2000$

Here, because of the symmetry of the Dynkin diagram, a weight $\left(a_{1}, a_{2}, a_{3}, a_{4}\right)$ and all those that are obtained from this by permuting $a_{1}, a_{2}$ and $a_{4}$ lead to representations of the same degree. For better readability, however, we include all of these weights in this table.

\begin{tabular}{|c|c|c|c|c|c|c|c|c|}
\hline deg & $\lambda$ & $p$ & deg & $\lambda$ & $p$ & deg & $\lambda$ & $p$ \\
\hline 1 & $(0000)$ & all & 322 & (1101) & 3 & 904 & (2101) & 5 \\
\hline 8 & (0001) & all & 350 & (1101) & $\neq 2,3$ & 1008 & (0104) & 7 \\
\hline 8 & $(0100)$ & all & 384 & (0111) & 3 & 1008 & $(0401)$ & 7 \\
\hline 8 & (1000) & all & 384 & (1011) & 3 & 1008 & (1004) & 7 \\
\hline 26 & (0010) & 2 & 384 & (1110) & 3 & 1008 & (1400) & 7 \\
\hline 28 & (0010) & $\neq 2$ & 518 & (0202) & 3 & 1008 & (4001) & 7 \\
\hline 35 & $(0002)$ & all & 518 & (2002) & 3 & 1008 & $(4100)$ & 7 \\
\hline 35 & $(0200)$ & all & 518 & $(2200)$ & 3 & 1144 & (1102) & 7 \\
\hline 35 & (2000) & all & 539 & (0012) & 5 & 1144 & (1201) & 7 \\
\hline 48 & (0101) & 2 & 539 & (0210) & 5 & 1144 & (2101) & 7 \\
\hline 48 & (1001) & 2 & 539 & (2010) & 5 & 1176 & (0021) & 3 \\
\hline 48 & (1100) & 2 & 560 & $(0005)$ & 7 & 1176 & (0120) & 3 \\
\hline 56 & (0101) & $\neq 2$ & 560 & $(0500)$ & 7 & 1176 & $(1020)$ & 3 \\
\hline 56 & (1001) & $\neq 2$ & 560 & $(5000)$ & 7 & 1256 & (0203) & 7 \\
\hline 56 & (1100) & $\neq 2$ & 567 & (0012) & $\neq 5$ & 1256 & $(0302)$ & 7 \\
\hline 104 & (0003) & 5 & 567 & $(0210)$ & $\neq 5$ & 1256 & (2003) & 7 \\
\hline 104 & (0011) & 3 & 567 & (2010) & $\neq 5$ & 1256 & $(2300)$ & 7 \\
\hline 104 & (0110) & 3 & 664 & (0013) & 5 & 1256 & (3002) & 7 \\
\hline 104 & (0300) & 5 & 664 & (0103) & 5 & 1256 & $(3200)$ & 7 \\
\hline 104 & (1010) & 3 & 664 & (0301) & 5 & 1296 & (1102) & $\neq 5,7$ \\
\hline 104 & (3000) & 5 & 664 & (0310) & 5 & 1296 & (1201) & $\neq 5,7$ \\
\hline 112 & (0003) & $\neq 5$ & 664 & (1003) & 5 & 1296 & (2101) & $\neq 5,7$ \\
\hline 112 & (0300) & $\neq 5$ & 664 & (1300) & 5 & 1322 & $(0022)$ & 5 \\
\hline 112 & (3000) & $\neq 5$ & 664 & (3001) & 5 & 1322 & $(0220)$ & 5 \\
\hline 152 & (0011) & 7 & 664 & (3010) & 5 & 1322 & (2020) & 5 \\
\hline 152 & (0110) & 7 & 664 & (3100) & 5 & 1351 & (0006) & 7 \\
\hline 152 & (1010) & 7 & 672 & $(0005)$ & $\neq 7$ & 1351 & $(0600)$ & 7 \\
\hline 160 & (0011) & $\neq 3,7$ & 672 & (0103) & $\neq 5$ & 1351 & $(6000)$ & 7 \\
\hline 160 & (0110) & $\neq 3,7$ & 672 & (0301) & $\neq 5$ & 1386 & (0006) & $\neq 7$ \\
\hline 160 & (1010) & $\neq 3,7$ & 672 & $(0500)$ & $\neq 7$ & 1386 & $(0600)$ & $\neq 7$ \\
\hline 168 & (0102) & 5 & 672 & (1003) & $\neq 5$ & 1386 & $(6000)$ & $\neq 7$ \\
\hline 168 & (0201) & 5 & 672 & (1300) & $\neq 5$ & 1400 & (0021) & $\neq 3$ \\
\hline 168 & (1002) & 5 & 672 & (3001) & $\neq 5$ & 1400 & (0120) & $\neq 3$ \\
\hline 168 & (1200) & 5 & 672 & $(3100)$ & $\neq 5$ & 1400 & (1020) & $\neq 3$ \\
\hline 168 & (2001) & 5 & 672 & $(5000)$ & $\neq 7$ & 1568 & (0013) & $\neq 5$ \\
\hline 168 & (2100) & 5 & 680 & (0111) & 5 & 1568 & (0310) & $\neq 5$ \\
\hline 195 & $(0020)$ & 3 & 680 & (1011) & 5 & 1568 & (3010) & $\neq 5$ \\
\hline 224 & (0102) & $\neq 5$ & 680 & (1110) & 5 & 1680 & (0104) & $\neq 7$ \\
\hline 224 & (0201) & $\neq 5$ & 784 & (0111) & 2 & 1680 & (0401) & $\neq 7$ \\
\hline 224 & (1002) & $\neq 5$ & 784 & (1011) & 2 & 1680 & (1004) & $\neq 7$ \\
\hline 224 & (1200) & $\neq 5$ & 784 & (1110) & 2 & 1680 & (1400) & $\neq 7$ \\
\hline 224 & (2001) & $\neq 5$ & 805 & (0202) & 5 & 1680 & (4001) & $\neq 7$ \\
\hline 224 & (2100) & $\neq 5$ & 805 & (2002) & 5 & 1680 & (4100) & $\neq 7$ \\
\hline 246 & (1101) & 2 & 805 & (2200) & 5 & 1841 & (1111) & 3 \\
\hline 293 & (0004) & 5 & 840 & (0111) & $\neq 2,3,5$ & 1896 & (0112) & 3 \\
\hline 293 & (0400) & 5 & 840 & (0202) & $\neq 3,5$ & 1896 & (0211) & 3 \\
\hline 293 & (4000) & 5 & 840 & (1011) & $\neq 2,3,5$ & 1896 & (1012) & 3 \\
\hline 294 & (0004) & $\neq 5$ & 840 & (1110) & $\neq 2,3,5$ & 1896 & (1210) & 3 \\
\hline 294 & (0400) & $\neq 5$ & 840 & (2002) & $\neq 3,5$ & 1896 & (2011) & 3 \\
\hline 294 & (4000) & $\neq 5$ & 840 & (2200) & $\neq 3,5$ & 1896 & (2110) & 3 \\
\hline 299 & (0020) & 7 & 904 & (1102) & 5 & 1925 & (0030) & all \\
\hline 300 & $(0020)$ & $\neq 3,7$ & 904 & (1201) & 5 & & & \\
\hline
\end{tabular}


Appendix A.42. Case $D_{5}, M=3000$

\begin{tabular}{|c|c|c|c|c|c|c|c|c|}
\hline deg & $\lambda$ & $p$ & deg & $\lambda$ & $p$ & deg & $\lambda$ & $p$ \\
\hline 1 & $(00000)$ & all & 576 & $(01002)$ & 3 & 1424 & $(12000)$ & 7 \\
\hline 10 & (00001) & all & 576 & (10002) & 3 & 1424 & (21000) & 7 \\
\hline 16 & (01000) & all & 606 & (00004) & 7 & 1440 & (12000) & $\neq 5,7$ \\
\hline 16 & (10000) & all & 656 & (01100) & 3 & 1440 & $(21000)$ & $\neq 5,7$ \\
\hline 44 & (00010) & 2 & 656 & (10100) & 3 & 1476 & (11001) & 5 \\
\hline 45 & (00010) & $\neq 2$ & 660 & (00004) & $\neq 7$ & 1608 & (11001) & 7 \\
\hline 53 & (00002) & 5 & 670 & (00101) & 2 & 1676 & $(00200)$ & 3 \\
\hline 54 & (00002) & $\neq 5$ & 672 & (03000) & $\neq 5$ & 1728 & (11001) & $\neq 2,5,7$ \\
\hline 100 & (00100) & 2 & 672 & (30000) & $\neq 5$ & 1772 & $(00005)$ & 7 \\
\hline 120 & $(00100)$ & $\neq 2$ & 720 & (01002) & $\neq 3$ & 1772 & $(00013)$ & 5 \\
\hline 126 & $(02000)$ & all & 720 & (10002) & $\neq 3$ & 1782 & $(00005)$ & $\neq 7$ \\
\hline 126 & (20000) & all & 770 & (00020) & $\neq 3$ & 1920 & (01003) & 7 \\
\hline 128 & (01001) & 5 & 840 & (02001) & 3 & 1920 & (10003) & 7 \\
\hline 128 & (10001) & 5 & 840 & (20001) & 3 & 2094 & (02010) & 5 \\
\hline 144 & (01001) & $\neq 5$ & 880 & (12000) & 5 & 2094 & (20010) & 5 \\
\hline 144 & (10001) & $\neq 5$ & 880 & (21000) & 5 & 2464 & (01011) & 3 \\
\hline 164 & (11000) & 2 & 945 & (00101) & $\neq 2$ & 2464 & (10011) & 3 \\
\hline 190 & (00011) & 3 & 1050 & (02001) & $\neq 3$ & 2640 & (01003) & $\neq 7$ \\
\hline 210 & (00003) & all & 1050 & (20001) & $\neq 3$ & 2640 & (10003) & $\neq 7$ \\
\hline 210 & (11000) & $\neq 2$ & 1056 & (01100) & 7 & 2650 & (00110) & 7 \\
\hline 320 & (00011) & $\neq 3$ & 1056 & (10100) & 7 & 2708 & (00110) & 2 \\
\hline 416 & (01010) & 2 & 1184 & (01100) & 2 & 2719 & (04000) & 5 \\
\hline 416 & (10010) & 2 & 1184 & (10100) & 2 & 2719 & (40000) & 5 \\
\hline 544 & (01010) & 3 & 1200 & (01100) & $\neq 2,3,7$ & 2772 & (04000) & $\neq 5$ \\
\hline 544 & (03000) & 5 & 1200 & (10100) & $\neq 2,3,7$ & 2772 & (40000) & $\neq 5$ \\
\hline 544 & (10010) & 3 & 1242 & (00110) & 3 & 2836 & (00102) & 5 \\
\hline 544 & (30000) & 5 & 1333 & (00012) & 5 & 2970 & (00110) & $\neq 2,3,7$ \\
\hline 559 & (00020) & 3 & 1341 & (00012) & 3 & 2976 & (01011) & 2 \\
\hline 560 & (01010) & $\neq 2,3$ & 1386 & (00012) & $\neq 3,5$ & 2976 & (10011) & 2 \\
\hline 560 & (10010) & $\neq 2,3$ & 1408 & (11001) & 2 & & & \\
\hline
\end{tabular}

Appendix A.43. Case $D_{6}, M=4000$

\begin{tabular}{rrr||rrr||rrr} 
deg & $\lambda$ & $p$ & $\operatorname{deg}$ & $\lambda$ & $p$ & $\operatorname{deg}$ & $\lambda$ & $p$ \\
\hline 1 & $(000000)$ & all & 462 & $(020000)$ & all & 1760 & $(010002)$ & 7 \\
12 & $(000001)$ & all & 462 & $(200000)$ & all & 1760 & $(100002)$ & 7 \\
32 & $(010000)$ & all & 495 & $(001000)$ & $\neq 2$ & 2013 & $(000101)$ & 5 \\
32 & $(100000)$ & all & 548 & $(000011)$ & 11 & 2079 & $(000101)$ & $\neq 2,5$ \\
64 & $(000010)$ & 2 & 560 & $(000011)$ & $\neq 3,11$ & 2112 & $(010002)$ & $\neq 7$ \\
66 & $(000010)$ & $\neq 2$ & 560 & $(110000)$ & 2 & 2112 & $(100002)$ & $\neq 7$ \\
76 & $(000002)$ & 3 & 792 & $(110000)$ & $\neq 2$ & 2740 & $(000110)$ & 3 \\
77 & $(000002)$ & $\neq 3$ & 1143 & $(000020)$ & 3 & 2784 & $(000012)$ & 3 \\
208 & $(000100)$ & 2 & 1286 & $(000004)$ & 7 & 2794 & $(000012)$ & 7 \\
220 & $(000100)$ & $\neq 2$ & 1287 & $(000004)$ & $\neq 7$ & 2848 & $(030000)$ & 5 \\
320 & $(010001)$ & 2 & 1376 & $(010010)$ & 5 & 2848 & $(300000)$ & 5 \\
320 & $(010001)$ & 3 & 1376 & $(100010)$ & 5 & 2860 & $(000012)$ & $\neq 3,7$ \\
320 & $(100001)$ & 2 & 1508 & $(000101)$ & 2 & 3200 & $(010100)$ & 2 \\
320 & $(100001)$ & 3 & 1561 & $(000020)$ & 5 & 3200 & $(100100)$ & 2 \\
340 & $(000003)$ & 7 & 1637 & $(000020)$ & 11 & 3808 & $(001001)$ & 2 \\
340 & $(000011)$ & 3 & 1638 & $(000020)$ & $\neq 3,5,11$ & 3960 & $(020001)$ & 7 \\
352 & $(000003)$ & $\neq 7$ & 1696 & $(010010)$ & 11 & 3960 & $(200001)$ & 7 \\
352 & $(010001)$ & $\neq 2,3$ & 1696 & $(100010)$ & 11 & 3992 & $(000013)$ & 5 \\
352 & $(100001)$ & $\neq 2,3$ & 1728 & $(010010)$ & $\neq 5,11$ & & & \\
364 & $(001000)$ & 2 & 1728 & $(100010)$ & $\neq 5,11$ & & &
\end{tabular}


Appendix A.44. Case $D_{7}, M=5000$

\begin{tabular}{rrr||rrr||rrr} 
deg & $\lambda$ & $p$ & deg & $\lambda$ & $p$ & deg & $\lambda$ & $p$ \\
\hline 1 & $(0000000)$ & all & 832 & $(0100001)$ & $\neq 7$ & 3003 & $(1100000)$ & $\neq 2$ \\
14 & $(0000001)$ & all & 832 & $(1000001)$ & $\neq 7$ & 3079 & $(0000020)$ & 13 \\
64 & $(0100000)$ & all & 882 & $(0000011)$ & 13 & 3080 & $(0000020)$ & $\neq 3,13$ \\
64 & $(1000000)$ & all & 896 & $(0000011)$ & $\neq 3,13$ & 3913 & $(0000101)$ & 3 \\
90 & $(0000010)$ & 2 & 910 & $(0001000)$ & 2 & 4004 & $(0000101)$ & $\neq 2,3$ \\
91 & $(0000010)$ & $\neq 2$ & 1001 & $(0001000)$ & $\neq 2$ & 4096 & $(0100010)$ & 2 \\
103 & $(0000002)$ & 7 & 1288 & $(0010000)$ & 2 & 4096 & $(0100010)$ & 3 \\
104 & $(0000002)$ & $\neq 7$ & 1716 & $(0200000)$ & all & 4096 & $(1000010)$ & 2 \\
336 & $(0000100)$ & 2 & 1716 & $(2000000)$ & all & 4096 & $(1000010)$ & 3 \\
364 & $(0000100)$ & $\neq 2$ & 1912 & $(1100000)$ & 2 & 4864 & $(0100010)$ & 13 \\
532 & $(0000011)$ & 3 & 1975 & $(0000020)$ & 3 & 4864 & $(1000010)$ & 13 \\
546 & $(0000003)$ & all & 2002 & $(0010000)$ & $\neq 2$ & 4928 & $(0100010)$ & $\neq 2,3,13$ \\
768 & $(0100001)$ & 7 & 2275 & $(0000004)$ & all & 4928 & $(1000010)$ & $\neq 2,3,13$ \\
768 & $(1000001)$ & 7 & 2884 & $(0000101)$ & 2 & & &
\end{tabular}

Appendix A.45. Case $D_{8}, M=10000$

\begin{tabular}{rrr||rrr||rrr} 
deg & $\lambda$ & $p$ & $\operatorname{deg}$ & $\lambda$ & $p$ & $\operatorname{deg}$ & $\lambda$ & $p$ \\
\hline 1 & $(00000000)$ & all & 1582 & $(00001000)$ & 2 & 5169 & $(00000020)$ & 7 \\
16 & $(00000001)$ & all & 1792 & $(01000001)$ & 2 & 5303 & $(00000020)$ & 5 \\
118 & $(00000010)$ & 2 & 1792 & $(10000001)$ & 2 & 5304 & $(00000020)$ & $\neq 3,5,7$ \\
120 & $(00000010)$ & $\neq 2$ & 1820 & $(00001000)$ & $\neq 2$ & 6435 & $(02000000)$ & all \\
128 & $(01000000)$ & all & 1920 & $(01000001)$ & $\neq 2$ & 6435 & $(20000000)$ & all \\
128 & $(10000000)$ & all & 1920 & $(10000001)$ & $\neq 2$ & 6528 & $(11000000)$ & 2 \\
135 & $(00000002)$ & all & 3483 & $(00000020)$ & 3 & 6900 & $(00000101)$ & 7 \\
544 & $(00000100)$ & 2 & 3605 & $(00000004)$ & 5 & 7020 & $(00000101)$ & $\neq 2,7$ \\
560 & $(00000100)$ & $\neq 2$ & 3740 & $(00000004)$ & $\neq 5$ & 8008 & $(00100000)$ & $\neq 2$ \\
768 & $(00000011)$ & 3 & 3808 & $(00010000)$ & 2 & 8805 & $(00000012)$ & 3 \\
800 & $(00000003)$ & all & 4368 & $(00010000)$ & $\neq 2$ & 8925 & $(00000012)$ & $\neq 3$ \\
1328 & $(00000011)$ & 5 & 4488 & $(00100000)$ & 2 & & & \\
1344 & $(00000011)$ & $\neq 3,5$ & 5066 & $(00000101)$ & 2 & & &
\end{tabular}

Appendix A.46. Case $D_{9}, M=15000$

\begin{tabular}{rrr||rrr||rrr} 
deg & $\lambda$ & $p$ & deg & $\lambda$ & $p$ & deg & $\lambda$ & $p$ \\
\hline 1 & $(000000000)$ & all & 1122 & $(000000003)$ & $\neq 5$ & 5814 & $(000000004)$ & $\neq 5,11$ \\
18 & $(000000001)$ & all & 1902 & $(000000011)$ & 17 & 6972 & $(000010000)$ & 2 \\
152 & $(000000010)$ & 2 & 1920 & $(000000011)$ & $\neq 3,17$ & 8226 & $(000000101)$ & 2 \\
153 & $(000000010)$ & $\neq 2$ & 2906 & $(000001000)$ & 2 & 8549 & $(000000020)$ & 17 \\
169 & $(000000002)$ & 3 & 3060 & $(000001000)$ & $\neq 2$ & 8550 & $(000000020)$ & $\neq 3,17$ \\
170 & $(000000002)$ & $\neq 3$ & 4096 & $(010000001)$ & 3 & 8568 & $(000010000)$ & $\neq 2$ \\
256 & $(010000000)$ & all & 4096 & $(100000001)$ & 3 & 11475 & $(000000101)$ & $\neq 2$ \\
256 & $(100000000)$ & all & 4352 & $(010000001)$ & $\neq 3$ & 14043 & $(000000012)$ & 3 \\
780 & $(000000100)$ & 2 & 4352 & $(100000001)$ & $\neq 3$ & 14059 & $(000000012)$ & 5 \\
816 & $(000000100)$ & $\neq 2$ & 5490 & $(000000020)$ & 3 & 14212 & $(000000012)$ & $\neq 3,5$ \\
1104 & $(000000003)$ & 5 & 5644 & $(000000004)$ & 11 & & & \\
1104 & $(000000011)$ & 3 & 5813 & $(000000004)$ & 5 & & &
\end{tabular}


Appendix A.47. Case $D_{10}, M=18000$

\begin{tabular}{rrr||rrr||rrr} 
deg & $\lambda$ & $p$ & deg & $\lambda$ & $p$ & deg & $\lambda$ & $p$ \\
\hline 1 & $(0000000000)$ & all & 1500 & $(0000000011)$ & 3 & 9216 & $(1000000001)$ & 2 \\
20 & $(0000000001)$ & all & 1520 & $(0000000003)$ & $\neq 11$ & 9216 & $(1000000001)$ & 5 \\
188 & $(0000000010)$ & 2 & 2620 & $(0000000011)$ & 19 & 9728 & $(0100000001)$ & $\neq 2,5$ \\
190 & $(0000000010)$ & $\neq 2$ & 2640 & $(0000000011)$ & $\neq 3,19$ & 9728 & $(1000000001)$ & $\neq 2,5$ \\
208 & $(0000000002)$ & 5 & 4466 & $(0000001000)$ & 2 & 12712 & $(0000000101)$ & 2 \\
209 & $(0000000002)$ & $\neq 5$ & 4845 & $(0000001000)$ & $\neq 2$ & 13089 & $(0000000020)$ & 19 \\
512 & $(0100000000)$ & all & 8036 & $(0000000020)$ & 3 & 13090 & $(0000000020)$ & $\neq 3,19$ \\
512 & $(1000000000)$ & all & 8644 & $(0000000004)$ & 11 & 14344 & $(0000010000)$ & 2 \\
1120 & $(0000000100)$ & 2 & 8645 & $(0000000004)$ & $\neq 11$ & 15504 & $(0000010000)$ & $\neq 2$ \\
1140 & $(0000000100)$ & $\neq 2$ & 9216 & $(0100000001)$ & 2 & 17575 & $(0000000101)$ & 3 \\
1500 & $(0000000003)$ & 11 & 9216 & $(0100000001)$ & 5 & 17765 & $(0000000101)$ & $\neq 2,3$
\end{tabular}

Appendix A.48. Case $D_{11}, M=20000$

\begin{tabular}{rrr||rrr||rrr} 
deg & $\lambda$ & $p$ & deg & $\lambda$ & $p$ & deg & $\lambda$ & $p$ \\
\hline 1 & $(00000000000)$ & all & 1496 & $(00000000100)$ & 2 & 11912 & $(00000000020)$ & 3 \\
22 & $(00000000001)$ & all & 1540 & $(00000000100)$ & $\neq 2$ & 12145 & $(00000000004)$ & 13 \\
230 & $(00000000010)$ & 2 & 1958 & $(00000000011)$ & 3 & 12397 & $(00000000004)$ & $\neq 13$ \\
231 & $(00000000010)$ & $\neq 2$ & 2002 & $(00000000003)$ & all & 18744 & $(00000000101)$ & 2 \\
251 & $(00000000002)$ & 11 & 3498 & $(00000000011)$ & 7 & 18976 & $(00000000020)$ & 5 \\
252 & $(00000000002)$ & $\neq 11$ & 3520 & $(00000000011)$ & $\neq 3,7$ & 19227 & $(00000000020)$ & 7 \\
1024 & $(01000000000)$ & all & 7084 & $(00000001000)$ & 2 & 19228 & $(00000000020)$ & $\neq 3,5,7$ \\
1024 & $(10000000000)$ & all & 7315 & $(00000001000)$ & $\neq 2$ & & &
\end{tabular}

Appendix A.49. Case $G_{2}, M=500$

\begin{tabular}{rrr||rrr||rrr} 
deg & $\lambda$ & $p$ & deg & $\lambda$ & $p$ & deg & $\lambda$ & $p$ \\
\hline 1 & $(00)$ & all & 97 & $(21)$ & 5 & 286 & $(21)$ & $\neq 3,5,7$ \\
6 & $(01)$ & 2 & 125 & $(12)$ & 11 & 295 & $(22)$ & 11 \\
7 & $(01)$ & $\neq 2$ & 148 & $(30)$ & 11 & 301 & $(05)$ & 13 \\
7 & $(10)$ & 3 & 155 & $(04)$ & 11 & 371 & $(05)$ & 11 \\
14 & $(10)$ & $\neq 3$ & 182 & $(04)$ & $\neq 11$ & 371 & $(13)$ & 5 \\
26 & $(02)$ & 7 & 189 & $(12)$ & $\neq 11$ & 378 & $(05)$ & $\neq 11,13$ \\
27 & $(02)$ & $\neq 7$ & 189 & $(21)$ & 3 & 434 & $(13)$ & 11 \\
27 & $(20)$ & 3 & 196 & $(30)$ & 5 & 448 & $(13)$ & $\neq 5,11,13$ \\
38 & $(11)$ & 7 & 244 & $(31)$ & 13 & 469 & $(14)$ & 5 \\
49 & $(11)$ & 3 & 248 & $(21)$ & 7 & 481 & $(22)$ & 7 \\
64 & $(11)$ & $\neq 3,7$ & 259 & $(13)$ & 13 & 483 & $(22)$ & 5 \\
77 & $(03)$ & all & 267 & $(40)$ & 7 & 489 & $(40)$ & 13 \\
77 & $(20)$ & $\neq 3$ & 273 & $(30)$ & $\neq 5,11$ & & &
\end{tabular}


Appendix A.50. Case $F_{4}, M=12000$

\begin{tabular}{rrr||rrr||rrr} 
deg & $\lambda$ & $p$ & $\operatorname{deg}$ & $\lambda$ & $p$ & $\operatorname{deg}$ & $\lambda$ & $p$ \\
\hline 1 & $(0000)$ & all & 755 & $(2000)$ & 7 & 5369 & $(1100)$ & 3 \\
25 & $(0001)$ & 3 & 1053 & $(1001)$ & $\neq 2$ & 6396 & $(0101)$ & 2 \\
26 & $(0001)$ & $\neq 3$ & 1053 & $(2000)$ & $\neq 7$ & 6396 & $(1010)$ & 2 \\
26 & $(1000)$ & 2 & 1222 & $(0100)$ & 3 & 6707 & $(1002)$ & 3 \\
52 & $(1000)$ & $\neq 2$ & 1274 & $(0100)$ & $\neq 2,3$ & 7371 & $(1010)$ & 3 \\
196 & $(0010)$ & 3 & 2404 & $(0011)$ & 3 & 8424 & $(1010)$ & $\neq 2,3,7$ \\
246 & $(0010)$ & 2 & 2651 & $(0003)$ & 7 & 9477 & $(2001)$ & 5 \\
246 & $(0100)$ & 2 & 2652 & $(0003)$ & $\neq 7$ & 10829 & $(1002)$ & $\neq 3$ \\
273 & $(0010)$ & $\neq 2,3$ & 2991 & $(0011)$ & 7 & 11102 & $(3000)$ & 5 \\
298 & $(0002)$ & 7 & 3773 & $(0011)$ & 13 & 11739 & $(0110)$ & 5 \\
323 & $(0002)$ & 13 & 4096 & $(0011)$ & $\neq 3,7,13$ & 11907 & $(0101)$ & 3 \\
324 & $(0002)$ & $\neq 7,13$ & 4096 & $(1100)$ & 2 & & & \\
676 & $(1001)$ & 2 & 4380 & $(1010)$ & 7 & & &
\end{tabular}

Appendix A.51. Case $E_{6}, M=50000$

\begin{tabular}{|c|c|c|c|c|c|c|c|c|}
\hline deg & $\lambda$ & $p$ & deg & $\lambda$ & $p$ & deg & $\lambda$ & $p$ \\
\hline 1 & $(000000)$ & all & 3003 & $(300000)$ & $\neq 5$ & 19305 & $(010002)$ & $\neq 3,7$ \\
\hline 27 & $(000001)$ & all & 5643 & $(001001)$ & 5 & 19305 & $(210000)$ & $\neq 3,7$ \\
\hline 27 & (100000) & all & 5643 & (100010) & 5 & 19305 & (400000) & $\neq 7$ \\
\hline 77 & $(010000)$ & 3 & 5746 & $(000011)$ & 5 & 20579 & (030000) & 5 \\
\hline 78 & $(010000)$ & $\neq 3$ & 5746 & (101000) & 5 & 23179 & (110001) & 5 \\
\hline 324 & $(000002)$ & 5 & 5824 & $(000011)$ & $\neq 3,5$ & 26244 & (000101) & 2 \\
\hline 324 & $(000010)$ & 2 & 5824 & (101000) & $\neq 3,5$ & 26244 & (100100) & 2 \\
\hline 324 & $(001000)$ & 2 & 5994 & (100002) & 7 & 28782 & (020001) & 3 \\
\hline 324 & (200000) & 5 & 5994 & (200001) & 7 & 28782 & (120000) & 3 \\
\hline 351 & $(000002)$ & $\neq 5$ & 6966 & $(001001)$ & 2 & 31668 & (110001) & 2 \\
\hline 351 & $(000010)$ & $\neq 2$ & 6966 & $(100010)$ & 2 & 32319 & (110001) & 3 \\
\hline 351 & $(001000)$ & $\neq 2$ & 7371 & (001001) & $\neq 2,5$ & 33021 & $(000020)$ & 5 \\
\hline 351 & (200000) & $\neq 5$ & 7371 & (100002) & 5 & 33021 & $(002000)$ & 5 \\
\hline 572 & (100001) & 2 & 7371 & (100010) & $\neq 2,5$ & 34099 & (110001) & 7 \\
\hline 572 & (100001) & 3 & 7371 & (200001) & 5 & 34398 & (000020) & $\neq 3,5$ \\
\hline 650 & (100001) & $\neq 2,3$ & 7722 & (100002) & $\neq 5,7$ & 34398 & $(002000)$ & $\neq 3,5$ \\
\hline 1377 & (010001) & 5 & 7722 & (200001) & $\neq 5,7$ & 34749 & (110001) & $\neq 2,3,5,7$ \\
\hline 1377 & (110000) & 5 & 9828 & (010010) & 2 & 35242 & (001010) & 2 \\
\hline 1701 & $(010001)$ & 13 & 9828 & $(011000)$ & 2 & 40831 & (010100) & 3 \\
\hline 1701 & (110000) & 13 & 11934 & $(010002)$ & 3 & 43758 & $(030000)$ & $\neq 5$ \\
\hline 1702 & $(000100)$ & 2 & 11934 & $(210000)$ & 3 & 44955 & $(020001)$ & 5 \\
\hline 1728 & (010001) & $\neq 5,13$ & 13311 & $(000004)$ & 7 & 44955 & (120000) & 5 \\
\hline 1728 & (110000) & $\neq 5,13$ & 13311 & $(400000)$ & 7 & 46332 & $(020001)$ & $\neq 3,5$ \\
\hline 2404 & (000011) & 3 & 15822 & (010010) & 3 & 46332 & (120000) & $\neq 3,5$ \\
\hline 2404 & (101000) & 3 & 15822 & $(011000)$ & 3 & 46656 & $(001002)$ & 3 \\
\hline 2429 & (020000) & 13 & 17550 & (010010) & $\neq 2,3$ & 46656 & (200010) & 3 \\
\hline 2430 & (020000) & $\neq 13$ & 17550 & (011000) & $\neq 2,3$ & 46683 & (000012) & 3 \\
\hline 2771 & $(000100)$ & 3 & 18225 & $(000020)$ & 3 & 46683 & $(000012)$ & 5 \\
\hline 2925 & $(000100)$ & $\neq 2,3$ & 18225 & $(002000)$ & 3 & 46683 & (201000) & 3 \\
\hline 3002 & $(000003)$ & 5 & 18954 & $(010002)$ & 7 & 46683 & (201000) & 5 \\
\hline 3002 & $(300000)$ & 5 & 18954 & $(210000)$ & 7 & & & \\
\hline 3003 & (000003) & $\neq 5$ & 19305 & (000004) & $\neq 7$ & & & \\
\hline
\end{tabular}


Small degree representations in defining characteristic

Appendix A.52. Case $E_{7}, M=100000$

\begin{tabular}{rrr||rrr||rrr} 
deg & $\lambda$ & $p$ & deg & $\lambda$ & $p$ & $\operatorname{deg}$ & $\lambda$ & $p$ \\
\hline 1 & $(0000000)$ & all & 6424 & $(1000001)$ & 19 & 27664 & $(0000100)$ & $\neq 2,3$ \\
56 & $(0000001)$ & all & 6480 & $(1000001)$ & $\neq 7,19$ & 30704 & $(0100001)$ & 2 \\
132 & $(1000000)$ & 2 & 7106 & $(0010000)$ & 2 & 30779 & $(0100001)$ & 3 \\
133 & $(1000000)$ & $\neq 2$ & 7370 & $(2000000)$ & 19 & 39217 & $(0100001)$ & 7 \\
856 & $(0100000)$ & 3 & 7371 & $(2000000)$ & $\neq 5,19$ & 40755 & $(0100001)$ & $\neq 2,3,7$ \\
912 & $(0100000)$ & $\neq 3$ & 8512 & $(0010000)$ & 3 & 44592 & $(0000011)$ & 11 \\
1274 & $(0000010)$ & 2 & 8645 & $(0010000)$ & $\neq 2,3$ & 50160 & $(0000011)$ & 2 \\
1330 & $(0000002)$ & 3 & 18752 & $(0000003)$ & 7 & 51072 & $(0000011)$ & $\neq 2,3,11$ \\
1463 & $(0000002)$ & $\neq 3$ & 21184 & $(0000100)$ & 2 & 57608 & $(1100000)$ & 5 \\
1538 & $(0000010)$ & 7 & 24264 & $(0000003)$ & 11 & 79704 & $(1100000)$ & 13 \\
1539 & $(0000010)$ & $\neq 2,7$ & 24264 & $(0000011)$ & 3 & 86128 & $(1100000)$ & 2 \\
5568 & $(1000001)$ & 7 & 24320 & $(0000003)$ & $\neq 7,11$ & 86184 & $(1100000)$ & $\neq 2,5,13$ \\
5832 & $(2000000)$ & 5 & 25896 & $(0000100)$ & 3 & & &
\end{tabular}

Appendix A.53. Case $E_{8}, M=100000$

\begin{tabular}{rrr||rrr||rrr} 
deg & $\lambda$ & $p$ & deg & $\lambda$ & $p$ & deg & $\lambda$ & $p$ \\
\hline 1 & $(00000000)$ & all & 23125 & $(00000002)$ & 7 & 30132 & $(00000010)$ & 3 \\
248 & $(00000001)$ & all & 26504 & $(00000010)$ & 2 & 30132 & $(00000010)$ & 5 \\
3626 & $(10000000)$ & 2 & 26999 & $(00000002)$ & 31 & 30380 & $(00000010)$ & $\neq 2,3,5$ \\
3875 & $(10000000)$ & $\neq 2$ & 27000 & $(00000002)$ & $\neq 7,31$ & & &
\end{tabular}

Frank Lübeck Frank. Luebeck@Math.RWTH-Aachen.De

RWTH Aachen

Lehrstuhl D für Mathematik

Templergraben 64

52062 Aachen

Germany

http: //www. math.rwth-aachen. de/ Frank. Luebeck/ 